\title{
Modeling and nonlinear control for air-breathing hypersonic vehicle with variable geometry inlet
}

\section{DOI:}

10.1016/j.ast.2017.04.024

\section{Document Version}

Accepted author manuscript

Link to publication record in Manchester Research Explorer

\section{Citation for published version (APA):}

Dou, L., Su, P., \& Ding, Z. (2017). Modeling and nonlinear control for air-breathing hypersonic vehicle with variable geometry inlet. Aerospace Science and Technology, 67, 422-432. https://doi.org/10.1016/j.ast.2017.04.024

\section{Published in:}

Aerospace Science and Technology

\section{Citing this paper}

Please note that where the full-text provided on Manchester Research Explorer is the Author Accepted Manuscript or Proof version this may differ from the final Published version. If citing, it is advised that you check and use the publisher's definitive version.

\section{General rights}

Copyright and moral rights for the publications made accessible in the Research Explorer are retained by the authors and/or other copyright owners and it is a condition of accessing publications that users recognise and abide by the legal requirements associated with these rights.

\section{Takedown policy}

If you believe that this document breaches copyright please refer to the University of Manchester's Takedown Procedures [http://man.ac.uk/04Y6Bo] or contact uml.scholarlycommunications@manchester.ac.uk providing relevant details, so we can investigate your claim.

\section{OPEN ACCESS}


Paper No. AESCTE_2016_290_R2

\title{
Modeling and nonlinear control for air-breathing hypersonic vehicle with variable geometry inlet
}

\author{
Liqian Dou ${ }^{\mathrm{a}}$, Peihua Su ${ }^{\mathrm{a}}$, Zhengtao Ding ${ }^{\mathrm{b}, *}$ \\ ${ }^{a}$ School of Electrical Engineering and Automation, Tianjin University Tianjin, 300072, China \\ ${ }^{b}$ School of Electrical and Electronic Engineering, The University of Manchester, Manchester, M13 9PL, U.K.
}

\begin{abstract}
This paper develops a control method for an air-breathing hypersonic vehicle with variable geometry inlet (AHV-VGI). For the AHV-VGI, a movable translating cowl is used to track the shock on lip conditions to capture enough air mass flow, which can ensure a more powerful thrust. Compared with traditional air-breathing hypersonic vehicle with fixed geometry inlet (AHV-FGI), this AHV-VGI extends the velocity range, which is favorable to the acceleration and maneuvering flight. However, the VGI causes the unknown changes of the aerodynamic forces, moment and the thrust in the meanwhile. Therefore, we firstly establish a longitudinal dynamic for AHV-VGI, which includes the uncertain changes induced by VGI. A conception of the optimal elongation distance of translating cowl is introduced, and its estimated value is obtained by curve fitted approximation. And then, the control process for AHV-VGI is divided into two subsystems. For each subsystem, a sliding mode controller is designed, and interval type-2 fuzzy logic systems (FLSs) are adopted to approximate nonlinear parts including the uncertain changes induced by VGI. Furthermore, uniformly stability of the whole system is proved by Lyapunov approach. Finally, simulation results demonstrate that AHV have a better control performance under the condition of VGI compared to the FGI.
\end{abstract}

Keywords: hypersonic vehicle, variable geometry inlet, movable translating cowl, type-2 fuzzy system, sliding mode control

\section{Introduction}

In recent years, air-breathing hypersonic vehicle (AHV) have attracted a great deal of attention, mainly because of reliable, affordable, routine access to space and prompt global reach [1][2][3]. Both of the technical advantages have commercial as well as military implications, as witnessed by the success of NASA's scramjet-powered X-43A and X-51A[4][5]. This type of vehicle has a unique design, incorporating a supersonic combustion scramjet engine located beneath the fuselage. This configuration can exhibit good performance in the range of Mach 4-7, but it causes severe aero-propulsion interactions and uncertainties, which result in highly nonlinear, strongly coupling, and fast time varying, with a great amount of parameter uncertainties as well as unknown external disturbances [6][7]. Therefore, the dynamics modeling and flight control design of AHV become one of the most challenging problems.

For modeling of hypersonic vehicle for control design, generally, there are three kinds of dynamics models in the open literature. The first model is Winged-Cone configurature called the generic hypersonic vehicle (GHV)[8]. The GHV model was developed to support NASA funded conceptual design studies of hypersonic flight vehicles. The aerodynamic characteristics of the vehicle were developed using a CFD-based study conducted

\footnotetext{
${ }^{*}$ Corresponding author: Zhengtao Ding

Email addresses: douliqian@t ju.edu.com (Liqian Dou), supeihua@tju.edu.cn (Peihua Su), zhengtao.ding@manchester.ac.uk (Zhengtao Ding )
}

jointly at NASA Langley and Rockwell International[9][10]. Based on the aerodynamic data, A model for the longitudinal dynamics of GHV was presented and dynamic inversion was adopted to design nonlinear robust controller[11][12]. The second dynamics model of hypersonic vehicle is a plane symmetrical configurature AHV. The model is the first analytical aeropropulsive/aeroelastic AHV model. In this model, a twodimensional hypersonic aerodynamic analysis using Newtonian theory was used, coupled with a one-dimensional aerothermodynamic analysis of the flow within a scramjet-type propulsion system[13]. Subsequently, some dynamic and robustness analysis were given based on this model[14][15]. The third model of AHV was developed by U.S. Air Force Research Laboratory (AFRL) engineers. This model captures many of the complex interactions between the propulsion system, the aerodynamics, and the structural dynamics, resulting in a more accurate and substantially more complex model[16]. Subsequently, for reducing the difficulty for nonlinear control of AHV, Parker established a rigid body/elastic body coupling model of hypersonic vehicle oriented for control. This model includes the coupling between the engine and flight mechanics, the coupling between compliant modes and rigid modes and the influence of these couplings on design of the control system [17]. Whatever the model is, steering the state to a desired trim condition along some reference trajectories is the main problem for AHV. Many of control methods and techniques have been applied to flight control design of AHV, such as linear quadratic regulator control[18], robust control[19], adaptive control[20], sequential loop closure controller design[21], slid- 
ing mode control (SMC)[22][23][24], disturbance-observerbased control[25], intelligent control algorithm[26][27], etc.

$\mathrm{AHV}$ is engineered to work within a wide speed range. However, in the context of the aforementioned literatures, AHV-FGI model was adopted for control design. Typically, the inlet of AHV-FGI are designed for the highest Mach number expected during the hypersonic flight, and at Mach numbers lower than the design value, the so-called spillage occurs and the air mass capture decreases[28]. That means that this kind of AHV-FGI is troubled by low air-capture ratio and large additional drag while operating at a low Mach number. Moreover, when the AHV-FGI is in the state acceleration or maneuvering flight, the shock wave would deviate from the scramjet lip, which lead to the scramjet engine could not get sufficient air flow, so that the thrust will be generated insufficiently[29]. In order to solve the problem, AHV-VGI was studied. NASA investigates a rotary lip VGI for the X-43A hypersonic aircraft[30], and Ref.[31] obtains the adjusting rules and performance variation of VGI in various conditions with numerical simulation method. The Space and Astronautical Science institution of Japan developed a variable geometry axisymmetric inlet for the ATREX engine[32]. Besides, the Russian scholar Kuranov investigated the Magneto Hydrodynamic controlled inlet[33] and an American scholar proposed a central adjustable cone inlet to improve the scramjet inlet performance in off-design conditions. These methods were proved to extend the operating range of flight Mach number.

In recent years, investigators have carried on many researches on VGI characteristic of AHV. In Ref.[34], a new methodology using gas dynamic relations has been developed to obtain optimal geometry of scramjet inlet at different Mach numbers. Ref.[31] compares the properties of three kinds of VGI using the low-order control-oriented model, and designs a kind of inlet used in a wide range of Mach numbers. Ref.[35] designs a 2-D hypersonic VGI with movable lip along the flow direction, carries out three dimensional CFD numerical simulations. Comparison of the aerodynamic characteristics was made between VGI and FGI. Ref.[36] investigates a rotary lip VGI adjustment program, presents a design method of highperformance VGI, and obtains the adjusting rules and performance variation of VGI in various conditions. Although there are many of researches on the configurature of AHV-VGI, the control system designs for AHV-VGI are investigated in the initial phase. The main reason is the complex structure of VGI system which is difficult to control well. A VGI scheme was proposed by [37]. By moving the movable cowl along the flow direction, the internal contraction ratio could be enlarged, and the propulsion efficiency could be improved. This VGI scheme is easy to operate, which only need to adjust the movable cowl to ensure that the shock wave can project right on the inlet lip. Thus, it provides a feasible idea for us to study the control design for AHV-VGI.

In this paper, we investigate the control problem for AHVVGI with movable cowl. A movable cowl can be used to track shock on lip conditons to capture off-design flow. By allowing the cowl to move with the changes of the shock position, proper mass flow can be taken into the engine without any flow spillage[29]. This VGI configuration can extend the velocity range and be favorable to the acceleration control of AHV[38]. However, the movement of cowl will induce the unknown changes of the aerodynamic forces and moment, especially, the thrust will be changed greatly. Ref.[39] shows that these changes are so large that cannot be regarded as small disturbances. Therefore, we will adopt interval type-2 fuzzy system to estimate the uncertain influences induced by VGI with movable cowl, and integrate the SMC method to achieve the tracking control goal. This paper is organized as follows. Section 2 states the basic principle of VGI with movable cowl, and establishes a longitudinal model of AHV-VGI. We discuss the basic definition of the optimal elongation distance of translating cowl and its effects on the thrust and aerodynamic. In Section 3, control design is divided into two parts, and velocity controller and altitude controller are designed using adaptive interval type-2 fuzzy SMC, respectively. Section 4 gives the simulation results in thress cases, and the acceleration control and altitude control are discussed in this part. The conclusion of the paper is drawn in Section 5.

\section{Problem Description}

\subsection{Model of AHV-VGI with movable translating cowl}

The three-dimensional structure of an AHV-VGI is shown in Fig. 1. The scramjet engine is located at the bottom of AHV. The translating cowl is in the forefront of the engine, which can be adjusted forward or backward with the requirements of flight control, thereby capture the proper air mass flow without any flow spillage.

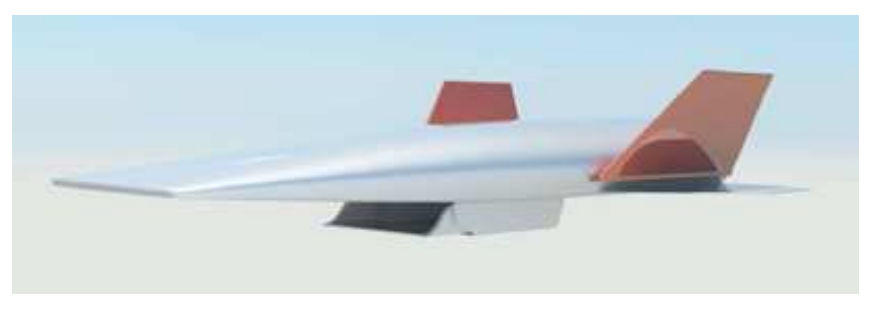

Fig. 1. The structure of AHV-VGI

As shown in Fig. 2, when the AHV operates in a cruise condition, the forebody oblique shock would occur.

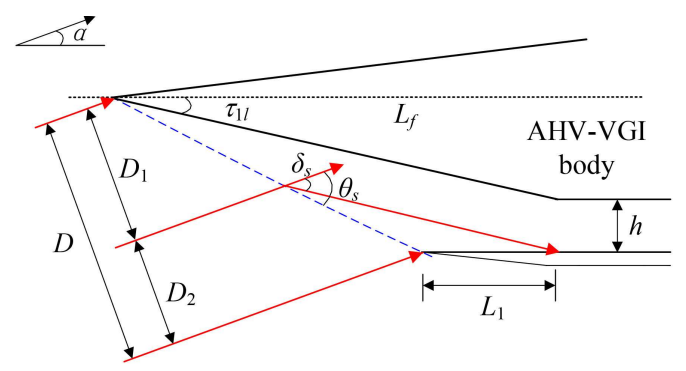

Fig. 2. Schematic of AHV with the translating cowl

The blue dashed line corresponds to the oblique shock wave, and $\theta_{s}$ is the shock wave angle. The red solid lines denote the 
free stream which hit against the oblique shock wave and then turn parallel to the lower forebody, $\delta_{s}=\alpha+\tau_{1 l}$ is the flow turn angle. Let $\left(M_{\infty}, P_{\infty}, T_{\infty}\right)$ denote the free-stream Mach, pressure, and temperature. Let $\lambda=1.4$ denotes the specific heat ratio for air - assumed constant in the model. The shock wave angle $\theta_{s}=\theta_{s}\left(M_{\infty}, \delta_{s}, \lambda\right)$ can be found as the middle root of the following shock angle polynomial:

$\sin ^{6} \theta_{s}+b \sin ^{4} \theta_{s}+c \sin ^{2} \theta_{s}+d=0$

where

$b=-\frac{M_{\infty}^{2}+2}{M_{\infty}^{2}}-\lambda \sin ^{2}\left(\delta_{s}\right)$

$c=\frac{2 M_{\infty}^{2}+1}{M_{\infty}^{4}}+\left[\frac{(\lambda+1)^{2}}{4}+\frac{\lambda-1}{M_{\infty}^{2}}\right] \sin ^{2}\left(\delta_{s}\right)$

$d=-\frac{\cos ^{2}\left(\delta_{s}\right)}{M_{\infty}^{4}}$

When the AHV operates in a low Mach number, the shock wave angle $\theta_{s}$ will be increased. Hence, if the cowl of the scramjet engine is fixed, the shock wave would not be sealed by the cowl. Consequently, that will cause the flow spillage (area $D_{2}$ ), and the scramjet engine would not obtain mass flow sufficiently ( $D_{1}$ is the actual captured area). In this case, if the cowl can be adjusted to the position $L_{1}$, the oblique shock wave can be sealed by the cowl again. Consequently, the inlet of scramjet will capture all mass flow of area $D=D_{1}+D_{2}$. The capture area can be calculated as

$D_{1}=\frac{h_{i} \sin \left(\theta_{s}\right) \cos \left(\tau_{1 l}\right)}{\sin \left(\theta_{s}-\alpha-\tau_{1 l}\right)}$

$D_{2}=\frac{h_{i}+L_{f} \tan \left(\tau_{1 l}\right) \sin \left(\theta_{s}\right)}{\sin \left(\theta_{s}-\alpha\right)}$

And then, the air mass flow through the engine is obtained by

$\dot{m}_{a}=P_{\infty} M_{\infty} \sqrt{\frac{\lambda}{R T_{\infty}}} D$

where the $\tau_{1 l}=6.2^{\circ}$ denotes the lower forebody angle, the height of engine inlet $h_{i}=3.5 \mathrm{ft}$, the forebody length $L_{f}=47 \mathrm{ft}, R$ is the gas constant for air. The proper distance of translating cowl $L_{1}$ is a function of the angle of attack (AOA) $\alpha$ and shock wave angle $\theta_{s}$ :

$L_{1}=L_{f}-\left(L_{f} \tan \tau_{1 l}+h_{i}\right) \cot \left(\theta_{s}-\alpha\right)$

The thrust produced by the engine is given by

$T=\dot{m}_{a}\left(v_{e}-v_{\infty}\right)+\left(P_{e}-P_{\infty}\right) A_{e}-\left(P_{1}-P_{\infty}\right) A_{1}$

where the $v_{e}$ is the exit flow velocity, $v_{\infty}$ is free stream flow velocity. $P_{1}$ is the pressure at the engine inlet entrance, $P_{e}$ is the pressure at the engine exit plane, $A_{1}$ and $A_{e}$ are the engine inlet area and exit area, respectively.
Definition 1: The optimal elongation distance of translating cowl $l$ is the position where the inlet of scramjet can capture all mass flow of area $D$, which can be obtained from (5).

Assumption 1: The translating cowl can be infinitely fast adjusted to the optimal elongation distance with the flight state of AHV (adjusting time is approximated to zero). That means the optimal elongation distance is the minimum extending distance of the translating cowl to achieve maximum free stream capture.

Obviously, the thrust of AHV is changed with the elongation distance $l$. By using the movable translating cowl, we can dramatically adjust the amount of air mass flow captured by inlet. It is helpful to reach a more powerful thrust. Besides, the aerodynamic forces and moments of the nacelle bottom will be influenced and ultimately the pitching moment of AHV will be changed. The above conclusions are detailed in the [39].

In this paper, the estimated value of the optimal elongation distance $l$ is presented by curve fitted approximation. From (1) and (5), we know that the $l$ is a function of Mach number $M a$ and AOA $\alpha$. The expression of fitting elongation distance $\hat{l}$ is shown in (7). The detailed parameters value of $C_{l}^{\alpha}, C_{l}^{\alpha^{2}}, C_{l}^{M a}$ and $C_{l}^{0}$ are found in the Appendix A.

$\hat{l} \approx C_{l}^{\alpha} \cdot \alpha+C_{l}^{\alpha^{2}} \cdot \alpha^{2}+C_{l}^{M a} \cdot M a+C_{l}^{0}$

Fig. 3 shows that the fitting error is very small. This error is within allowable range from the perspective of control design.

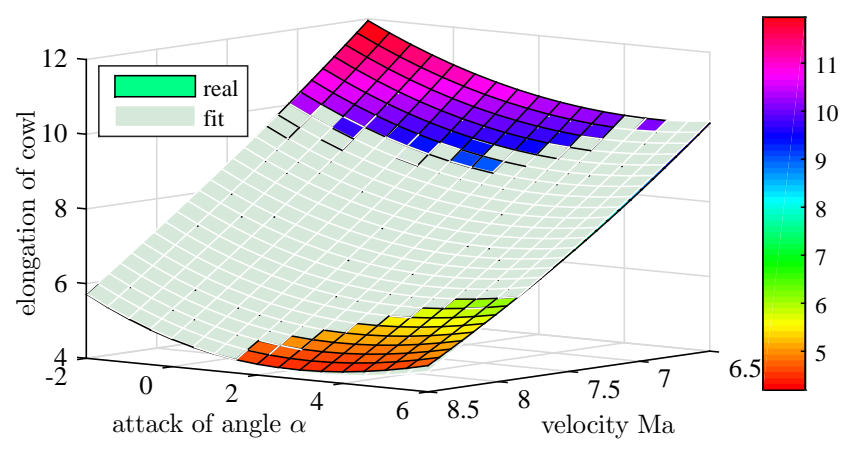

Fig. 3. The optimal elongation length of translating cowl

\subsection{The longitudinal model of VGI-AHV}

Differ from the longitudinal model of AHV-FGI, the aerodynamic characteristics in AHV-VGI model change with the elongation distance $l$. This change is embodied in the variations of aerodynamic forces: lift $L$, $\operatorname{drag} D$, thrust $T$ and pitching moment $M$. The dynamic model adopted in this study is on the basis of Bolender and Doman for the longitudinal rigid model of AHV[37]. By the least squares numerical fitting for AHVVGI, we obtain the analytical expressions of aerodynamic. The longitudinal dynamics of the AHV-VGI can be described by a set of differential equations for velocity $V$, altitude $h$, flight path angle (FPA) $\gamma, \mathrm{AOA} \alpha$, and pitching rate $Q$ as 
$\dot{V}=(T \cos \alpha-D) / m-g \sin \gamma$

$\dot{h}=V \sin \gamma$

$\dot{\gamma}=(T \sin \alpha+L) /(m V)-g \cos \gamma / V$

$\dot{\alpha}=Q-\dot{\gamma}$

$\dot{Q}=M / I_{y y}$

where $m, I_{y y}, g$ are the mass, moment of inertia, and acceleration of gravity respectively. In particular, $L, D, T, M$ are lift, drag, thrust, and pitching moment of AHV-VGI, which can be approximated by

$$
\begin{aligned}
& L \approx \bar{q} S\left(C_{L}+C_{L}^{l} \cdot \hat{l}\right) \\
& D \approx \bar{q} S\left(C_{D}+C_{D}^{l} \cdot \hat{l}\right) \\
& T \approx \bar{q}\left(C_{T}+C_{T}^{\phi} \cdot \phi+C_{T}^{l} \cdot \hat{l}\right) \\
& M \approx \bar{q} S \bar{c}\left(C_{M}+C_{M}^{l} \cdot \hat{l}\right)+z_{T} T
\end{aligned}
$$

where, $C_{L}, C_{D}, C_{M}$ and $C_{T}$ are the aerodynamic lift coefficients, drag coefficients, pitching moment coefficients and thrust coefficients. $C_{L}^{l}, C_{D}^{l}, C_{M}^{l}$ and $C_{T}^{l}$ are the disturbance coefficients that introduced by the movable translating cowl. $\hat{l}$ is the estimated value of elongation distance of translating cowl; $\bar{q}=1 / 2 \rho V^{2}$ denotes the dynamic pressure; $\rho, S, \bar{c}, z_{T}$ are air density, reference area, aerodynamic chord, and thrust moment arm respectively. The expressions of aerodynamic coefficients are shown in (14). The detailed values of aerodynamic coefficients are given in the Appendix A.

$$
\begin{aligned}
C_{L}= & C_{L}^{\alpha} \alpha+C_{L}^{M a} M a+C_{L}^{\delta_{e}} \delta_{e}+C_{L}^{0} \\
C_{D}= & C_{D}^{\alpha} \alpha+C_{D}^{\alpha^{2}} \alpha^{2}+C_{D}^{\delta_{e}} \delta_{e}+C_{D}^{\delta_{e}^{2}} \delta_{e}^{2}+C_{D}^{\alpha \delta_{e}} \alpha \delta_{e} \\
& +C_{D}^{M a} M a+C_{D}^{0} \\
C_{T}= & C_{T}^{\alpha} \alpha+C_{T}^{M a} M a+C_{T}^{0} \\
C_{T}^{\phi}= & C_{T}^{\phi \alpha} \alpha+C_{T}^{\phi M a} M a+C_{T}^{\phi} \\
C_{M}= & C_{M}^{\alpha} \alpha+C_{M}^{M a} M a+C_{M}^{\delta_{e}} \delta_{e}+C_{M}^{\phi} \phi+C_{M}^{0} \\
C_{L}^{l}= & C_{L, l}^{\alpha} \alpha+C_{L, l}^{M a} M a+C_{L, l}^{0} \\
C_{D}^{l}= & C_{D, l}^{\alpha} \alpha+C_{D, l}^{M a} M a+C_{D, l}^{0} \\
C_{T}^{l}= & C_{T, l}^{\alpha} \alpha+C_{T, l}^{M a} M a+C_{T, l}^{0} \\
C_{M}^{l}= & C_{M, l}^{\alpha} \alpha+C_{M, l}^{M a} M a+C_{M, l}^{0}
\end{aligned}
$$

The control inputs are the normalized fuel equivalence ratio (FER) $\phi$ and the elevator deflection $\delta_{e}$. The outputs are the velocity $V$ and altitude $h$. The instructions of velocity and altitude are denoted by $V_{d}$ and $h_{d}$ respectively.

\subsection{Control scheme}

The performance of AHV propulsion system is greatly improved at a very low cost by using movable translating cowl. However, the introduction of the movable cowl increases the nonlinearity and uncertainty of AHV dynamic model. As a result, the stability of the AHV control system becomes worse. From (7), we know that the elongation distance $l$ is a constant or varies in very small range when the AHV operates in a cruise condition, which can be taken as a small disturbance; However, when the AHV is in the state of acceleration or maneuvering flight, the aerodynamic forces change greatly, which easily causes the AHV system unstable. At this point, we need to quantitatively estimate the impacts of translating cowl on aerodynamic forces. In this paper, an adaptive interval type-2 fuzzy SMC method is proposed.

\section{Control System Design}

\subsection{Description of interval type-2 FLS}

In this paper, we adopt type-2 fuzzy sets. The concept of type- 2 fuzzy sets was first introduced by Zadeh as an extension of the type-1 fuzzy sets. The membership functions of type- 2 fuzzy sets are three-dimensional, which provide an additional degree of freedom. Compared to type-1 fuzzy logic system, the ability of type- 2 fuzzy logic system to deal with uncertainties is greatly increased. A type-2 fuzzy set in the universal set $X$ is denoted as $\tilde{A}$ which is characterized by a type- 2 membership function $\mu_{\tilde{A}}(x, u)$ :

$\tilde{A}=\int_{x \in X} \int_{u \in J_{x}} \frac{\mu_{\tilde{A}}(x, u)}{(x, u)}$

where, $x \in X$ is the primary variable and $u \subseteq J_{x} \subseteq[0,1]$ is the secondary variable.

The interval type- 2 fuzzy sets are characterized by interval type- 2 membership functions in which the secondary membership grades are equal to 1 . It is described as

$\tilde{A}=\int_{x \in X} \int_{u \in J_{x}} \frac{1}{(x, u)}=\int_{x \in X} \frac{\int_{u \in J_{x}} 1 / u}{x}$

Obviously, the interval type-2 fuzzy logic system is computationally far less intensive than the type-2 fuzzy logic system and thus better suitable for nonlinear complex control systems.

The structure of interval type-2 fuzzy logic system is shown in Fig. 4. There are five parts: fuzzifier, rule bases, inference engine, type-reducer, and defuzzifier.

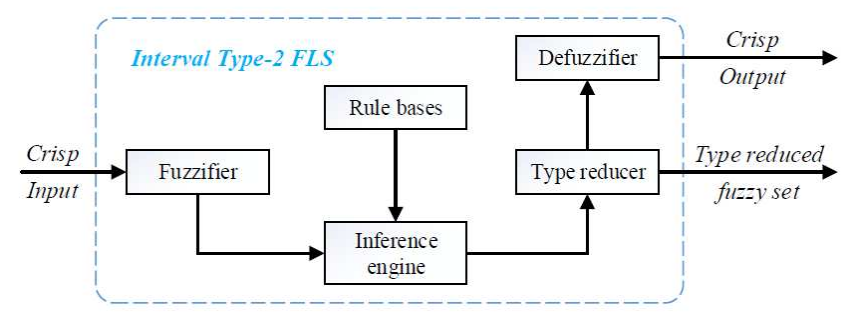

Fig. 4. The interval type-2 fuzzy logic system

Using the singleton fuzzifier, product inference engine, and the center of sets type reducer. Then, the output of the type reducer is an interval type-1 fuzzy set, which can be described by its two end points $y_{l}$ and $y_{r} . y_{l}$ and $y_{r}$ can be calculated through Karnik-Mendel algorithm [40]. We defuzzify the interval set 
by using the average of $y_{l}$ and $y_{r}$, hence, the defuzzified crisp output becomes

$y_{f u z z 2}=\frac{1}{2}\left(y_{l}+y_{r}\right)=\frac{1}{2}\left(\theta_{l}^{T} \xi_{l}+\theta_{r}^{T} \xi_{r}\right)=\theta^{T} \xi$

where, $y_{l}=\theta_{l}^{T} \xi_{l}, y_{r}=\theta_{r}^{T} \xi_{r} . \theta_{l}, \theta_{r}$ and $\xi_{l}, \xi_{r}$ denote respectively the left and right adjustable parameter vectors and their associated regressive matrices, and $\theta^{T}=1 / 2\left[\begin{array}{ll}\theta_{l}^{T} & \theta_{r}^{T}\end{array}\right], \xi^{T}=\left[\begin{array}{ll}\xi_{l}^{T} & \xi_{r}^{T}\end{array}\right]$.

Based on the universal approximation theorem, the interval fuzzy logic system (17) is a universal approximator in the sense that given any real continuous function $f: R^{n} \rightarrow R$ in a compact set $\Omega \in R^{n}$, and for any $\varepsilon>0$, there exists an interval type2 fuzzy logic system (17) such that $\sup _{x \in \Omega}\left|\theta^{T} \xi(x)-f(x)\right|<\varepsilon$. Then the function $f(x)$ can be expressed as

$f(x)=\theta^{* T} \xi(x)+\varepsilon, \forall x \in \Omega \in R^{n}$

where $\theta^{*}$ is the optimal parameter vector of $\theta$ and defined as

$\theta^{*}=\arg \min _{\theta \in R^{n}}\left\{\sup _{x \in \Omega}\left|\theta^{T} \xi(x)-f(x)\right|\right\}$

The adaptive interval type-2 fuzzy logic system is used to identify the nonlinear complex terms in the control system design.

\subsection{Control system structure}

The overall control system of AHV-VGI can be seen in Fig. 5. The inputs of adaptive interval type-2 fuzzy sliding mode controller are velocity deviation $e_{V}$, altitude deviation $e_{h}$, five rigid states $[V, h, \gamma, \alpha, Q]$, two control states $\left[\phi, \delta_{e}\right]$, and the estimate value of elongation distance $\hat{l}$.

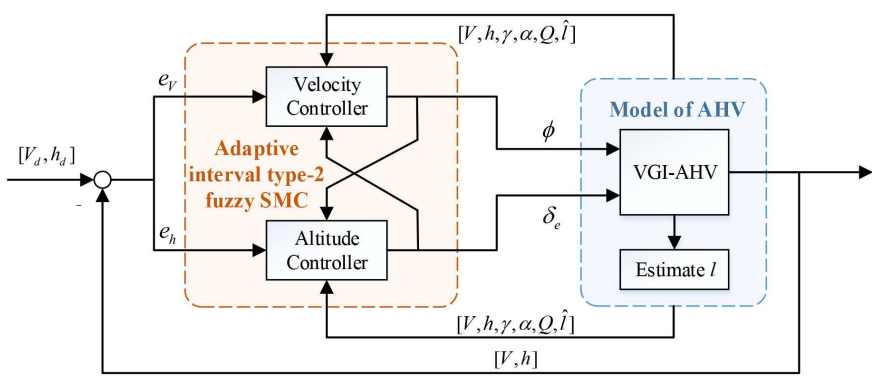

Fig. 5. The block diagram of AHV control system

\subsection{Design of adaptive interval type-2 fuzzy controller}

In order to apply the backstepping method, the equations of motion are transformed into a strict feedback form. The rigid states $x=[V, h, \gamma, \alpha, Q]^{T}$ and control inputs $u=\left[\phi, \delta_{e}\right]^{T}$ are assumed to be available for measurement. The strict feedback equations are presented as

$$
\begin{aligned}
& \dot{V}=g_{V} \phi+f_{V} \\
& \dot{h}=V \sin \gamma \\
& \dot{\gamma}=g_{1} \alpha+f_{1} \\
& \dot{\alpha}=g_{2} Q+f_{2} \\
& \dot{Q}=g_{3} \delta_{e}+f_{3}
\end{aligned}
$$

where

$$
\begin{aligned}
g_{V}= & \bar{q} C_{T}^{\phi} \cos \alpha / m \\
f_{V}= & \bar{q}\left[C_{T} \cos \alpha-S\left(C_{D}^{\alpha} \alpha+C_{D}^{\alpha^{2}} \alpha^{2}+C_{D}^{M a} M a+C_{D}^{0}\right)\right] / m \\
& -g \sin \gamma+f_{V}^{l} \\
g_{1}= & \bar{q} S C_{L}^{\alpha} /(m V) \\
f_{1}= & \bar{q}\left[S\left(C_{L}^{M a} M a+C_{L}^{0}\right)\right] /(m V)-g \cos \gamma / V+f_{\gamma}^{l} \\
g_{2}= & 1 \\
f_{2}= & -\bar{q}\left(C_{T} \sin \alpha+S C_{L}\right) /(m V)+f_{\alpha}^{l} \\
g_{3}= & \bar{q} S \bar{c} C_{M}^{\delta_{e}} / I_{y y} \\
f_{3}= & \bar{q}\left[S \bar{c}\left(C_{M}^{\alpha} \cdot \alpha+C_{M}^{M a} \cdot M a+C_{M}^{0}\right)+z_{T} C_{T}\right] / I_{y y}+f_{Q}^{l}
\end{aligned}
$$

where $f_{V}^{l}, f_{\gamma}^{l}, f_{\alpha}^{l}, f_{Q}^{l}$ are the uncertain disturbances introduced by movable cowl. The detailed approximate expressions of these functions are given in the Appendix B.

Assumption 2: The functions $g_{i}$, and its differentials $\dot{g}_{i}$ are bounded. Let $0<\underline{g}_{i} \leq g_{i} \leq \bar{g}_{i}$, and $0<\left|\dot{g}_{i}\right| \leq g_{i}^{d}, i=V, 1,2,3$. where $\bar{g}_{i}, g_{i}^{d}$ are the upper bounds, $\underline{g}_{i}$ are lower bounds.

Remark 1: Assumption2 is to ensure the control signals are nonsingular and bounded.

\subsubsection{Velocity controller design}

The velocity controller which shows in Fig. 5 outputs a control state FER $\phi$. The detailed structure can be seen in Fig. 6, where $Z_{V}=\left[\gamma, \alpha, \delta_{e}, V, \hat{l}\right]$. The velocity controller is designed as follows.

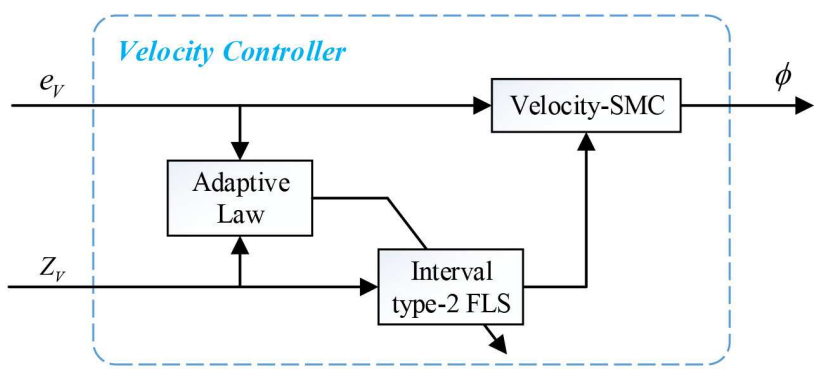

Fig. 6. Structure diagram of velocity subsystem

Define the velocity sliding mode surface: $S_{V}=e_{V}=V-V_{d}$, and its derivative is

$$
\begin{aligned}
\dot{S}_{V} & =\dot{V}-\dot{V}_{d}=g_{V} \phi+f_{V}-\dot{V}_{d} \\
& =g_{V}\left[\phi+g_{V}^{-1} f_{V}-g_{V}^{-1} \dot{V}_{d}\right]
\end{aligned}
$$

where the complex nonlinear functions $g_{V}^{-1} f_{V}$ and $g_{V}^{-1}$ are respectively estimated by two adaptive interval type-2 FLSs as

$$
\begin{aligned}
& g_{V}^{-1} f_{V}=\left(\theta_{V l}^{* T} \xi_{V l}+\theta_{V r}^{* T} \xi_{V r}\right) / 2+\varepsilon_{V 1} \\
& g_{V}^{-1}=\left(\lambda_{V l}^{* T} \psi_{V l}+\lambda_{V r}^{* T} \psi_{V r}\right) / 2+\varepsilon_{V 2}
\end{aligned}
$$

where $\varepsilon_{V 1}$ and $\varepsilon_{V 1}$ are approximation errors. 
Chose the reaching law $\dot{S}_{V}=-k_{V 1} S_{V}-k_{V 2} S_{V} /\left(\left|S_{V}\right|+\delta\right)$, then the control signal FER $\phi$ can be determined as

$$
\begin{aligned}
\phi=- & k_{V 1} S_{V}-k_{V 2} S_{V} /\left(\left|S_{V}\right|+\delta\right)-\left(\hat{\theta}_{V l}^{T} \xi_{V l}+\hat{\theta}_{V r}^{T} \xi_{V r}\right) / 2 \\
& +\dot{V}_{d}\left(\hat{\lambda}_{V l}^{T} \psi_{V l}+\hat{\lambda}_{V r}^{T} \psi_{V r}\right) / 2
\end{aligned}
$$

where $\delta$ is a very small positive constant; $k_{V 1}$ and $k_{V 2}$ are positive designed contants; $\hat{\theta}_{V l}, \hat{\theta}_{V r}, \hat{\lambda}_{V l}$ and $\hat{\lambda}_{V r}$ are estimated values of $\theta_{V l}^{*}, \theta_{V r}^{*}, \lambda_{V l}^{*}$ and $\lambda_{V r}^{*}$ respectively.

Remark 2: The continuous function $S_{V} /\left(\left|S_{V}\right|+\delta\right)$ is sliding mode switch item, which can ensure the control law is smooth and derivable.

$$
\begin{aligned}
& \dot{\hat{\theta}}_{V l}=\gamma_{V l}\left(\xi_{V l} S_{V} / 2-c_{V l} \hat{\theta}_{V l}\right) \\
& \dot{\hat{\theta}}_{V r}=\gamma_{V r}\left(\xi_{V r} S_{V} / 2-c_{V r} \hat{\theta}_{V r}\right) \\
& \dot{\hat{\lambda}}_{V l}=\tau_{V l}\left(-\psi_{V l} S_{V} \dot{V}_{d} / 2-d_{V l} \hat{\lambda}_{V l}\right) \\
& \dot{\hat{\lambda}}_{V r}=\tau_{V r}\left(-\psi_{V r} S_{V} \dot{V}_{d} / 2-d_{V r} \hat{\lambda}_{V r}\right)
\end{aligned}
$$

where $\gamma_{V l}, c_{V l}, \gamma_{V r}, c_{V r}, \tau_{V l}, d_{V l}, \tau_{V r}$ and $d_{V l}$ are positive designed constants.

\subsubsection{Altitude controller design}

Eq.(21) shows that the altitude $h$ and FPA $\gamma$ have a one to one relationship, so we transform the altitude instruction $h_{d}$ into FPA instruction $\gamma_{d}$ :

$\gamma_{d}=\arcsin \left[-k_{h}\left(h-h_{d}\right) / V\right]$

The altitude controller which shows in Fig. 5 outputs a control state elevator deflection $\delta_{e}$. The detailed structure can be seen in Fig. 7 , where $Z_{\gamma}=[\gamma, \phi, V, \hat{l}], Z_{\alpha}=[\gamma, \alpha, \phi, V, \hat{l}]$ and $Z_{Q}=[\alpha, \phi, V, \hat{l}]$. The design of altitude controller can be divided into three steps: the FPA controller, the AOA controller, and the pitch angle rate controller. For each controller, adaptive interval type-2 FLSs are designed to approximate the complex nonlinear parts in its sliding mode control law. The structure of every subsystem is similar to the velocity subsystem which shown in Fig. 6. The first-order filter is introduced to eliminate the "explosion of terms" problem.

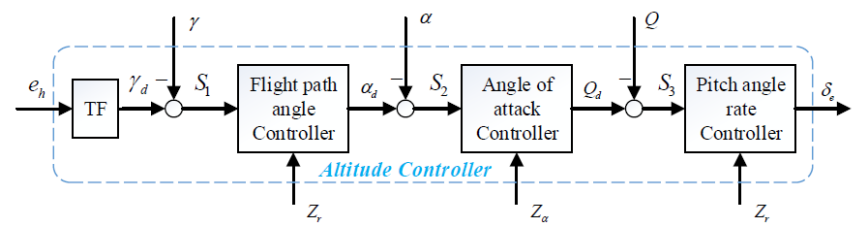

Fig. 7. Structure diagram of altitude subsystem

\section{Step 1:}

Define the sliding mode surface: $S_{1}=\gamma-\gamma_{d}$. The time derivative of $S_{1}$ is

$$
\begin{aligned}
\dot{S}_{1} & =\dot{\gamma}-\dot{\gamma}_{d}=g_{1} \alpha+f_{1}-\dot{\gamma}_{d} \\
& =g_{1}\left[\alpha+g_{1}^{-1} f_{1}-g_{1}^{-1} \dot{\gamma}_{d}\right]
\end{aligned}
$$

Since the functions $f_{1}$ and $g_{1}$ are unknown nonlinear terms. The interval type-2 FLSs are used to approximate the functions $g_{1}^{-1} f_{1}$ and $g_{1}^{-1}$ as

$g_{1}^{-1} f_{1}=\left(\theta_{1 l}^{* T} \xi_{1 l}+\theta_{1 r}^{* T} \xi_{1 r}\right) / 2+\varepsilon_{11}$

$g_{1}^{-1}=\left(\lambda_{1 l}^{* T} \psi_{1 l}+\lambda_{1 r}^{* T} \psi_{1 r}\right) / 2+\varepsilon_{12}$

where $\varepsilon_{11}$ and $\varepsilon_{12}$ are approximation errors. Choose the reaching law $\dot{S}_{1}=-k_{11} S_{1}-k_{12} S_{1} /\left(\left|S_{1}\right|+\delta\right)$. Then, the virtual control input is designed as

$$
\begin{aligned}
\bar{\alpha}_{d}= & -k_{11} S_{1}-k_{12} S_{1} /\left(\left|S_{1}\right|+\delta\right)-\left(\hat{\theta}_{1 l}^{T} \xi_{1 l}+\hat{\theta}_{1 r}^{T} \xi_{1 r}\right) / 2 \\
& +\dot{\gamma}_{d}\left(\hat{\lambda}_{1 l}^{T} \psi_{1 l}+\hat{\lambda}_{1 l}^{T} \psi_{1 l}\right) / 2
\end{aligned}
$$

where $k_{11}$ and $k_{12}$ is positive constants; $\hat{\theta}_{1 l}, \hat{\theta}_{1 r}, \hat{\lambda}_{1 l}, \hat{\lambda}_{1 r}$ are estimated values of $\theta_{1 l}^{*}, \theta_{1 r}^{*}, \lambda_{1 l}^{*}, \lambda_{1 r}^{*}$ respectively. Let $\bar{\alpha}_{d}$ pass through a first-order filter, a new state variable $\alpha_{d}$ can be obtained

$\kappa_{2} \dot{\alpha}_{d}+\alpha_{d}=\bar{\alpha}_{d} \quad \alpha_{d}(0)=\bar{\alpha}_{d}(0)$

The adaptive update laws of $\hat{\theta}_{1 l}, \hat{\theta}_{1 r}, \hat{\lambda}_{1 l}$ and $\hat{\lambda}_{1 r}$ can be designed as

$$
\begin{aligned}
& \dot{\hat{\theta}}_{1 l}=\gamma_{1 l}\left(\xi_{1 l} S_{1} / 2-c_{1 l} \hat{\theta}_{1 l}\right) \\
& \dot{\hat{\theta}}_{1 r}=\gamma_{1 r}\left(\xi_{1 r} S_{1} / 2-c_{1 r} \hat{\theta}_{1 r}\right) \\
& \dot{\hat{\lambda}}_{1 l}=\tau_{1 l}\left(-\psi_{1 l} S_{1} \dot{\gamma}_{d} / 2-d_{1 l} \hat{\lambda}_{1 l}\right) \\
& \dot{\hat{\lambda}}_{1 r}=\tau_{1 r}\left(-\psi_{1 r} S_{1} \dot{\gamma}_{d} / 2-d_{1 r} \hat{\lambda}_{1 r}\right)
\end{aligned}
$$

\section{Step 2:}

Define the sliding mode surface of AOA: $S_{2}=\alpha-\alpha_{d}$. The time derivative of $S_{2}$ is

$\dot{S}_{2}=\dot{\alpha}-\dot{\alpha}_{d}=Q+f_{2}-\dot{\alpha}_{d}$

we adopt an interval type- 2 fuzzy system to approximate the nonlinear function $f_{2}$ as follows.

$f_{2}=\left(\theta_{2 l}^{* T} \xi_{2 l}+\theta_{2 r}^{* T} \xi_{2 r}\right) / 2+\varepsilon_{2}$

where $\varepsilon_{2}$ is approximation error. Choose the reaching law $\dot{S}_{2}=$ $-k_{21} S_{2}-k_{22} S_{2} /\left(\left|S_{2}\right|+\delta\right)$. Then, The virtual control input is designed as

$$
\begin{aligned}
\bar{Q}_{d}= & -S_{1}-k_{21} S_{2}-k_{22} S_{2} /\left(\left|S_{2}\right|+\delta\right) \\
& -\left(\hat{\theta}_{2 l}^{T} \xi_{2 l}+\hat{\theta}_{2 r}^{T} \xi_{2 r}\right) / 2+\dot{\alpha}_{d}
\end{aligned}
$$

where $k_{21}$ and $k_{22}$ are positive constants; $\hat{\theta}_{2 l}$ and $\hat{\theta}_{2 r}$ are estimated values of $\theta_{2 l}^{*}$ and $\theta_{2 r}^{*}$ respectively. Let $\bar{Q}_{d}$ pass through a first-order filter, a new state variable $Q_{d}$ can be obtained

$\kappa_{3} \dot{Q}_{d}+Q_{d}=\bar{Q}_{d} \quad Q_{d}(0)=\bar{Q}_{d}(0)$

Design the adaptive update laws of $\hat{\theta}_{2 l}$ and $\hat{\theta}_{2 r}$ as

$$
\begin{aligned}
& \dot{\hat{\theta}}_{2 l}=\gamma_{2 l}\left(\xi_{2 l} S_{2} / 2-c_{2 l} \hat{\theta}_{2 l}\right) \\
& \dot{\hat{\theta}}_{2 r}=\gamma_{2 r}\left(\xi_{2 r} S_{2} / 2-c_{2 r} \hat{\theta}_{2 r}\right)
\end{aligned}
$$




\section{Step 3:}

Define the sliding mode surface: $S_{3}=Q-Q_{d}$. The time derivative of $S_{3}$ is

$$
\begin{aligned}
\dot{S}_{3} & =\dot{Q}-\dot{Q}_{d}=g_{3} \delta_{e}+f_{3}-\dot{Q}_{d} \\
& =g_{3}\left[\delta_{e}+g_{3}^{-1} f_{3}-g_{3}^{-1} \dot{Q}_{d}\right]
\end{aligned}
$$

Interval type-2 FLSs are used to approximate the functions $g_{3}^{-1} f_{3}$ and $g_{3}^{-1}$ as

$g_{3}^{-1} f_{3}=\left(\theta_{3 l}^{* T} \xi_{3 l}+\theta_{3 r}^{* T} \xi_{3 r}\right) / 2+\varepsilon_{31}$

$g_{3}^{-1}=\left(\lambda_{3 l}^{* T} \psi_{3 l}+\lambda_{3 r}^{* T} \psi_{3 r}\right) / 2+\varepsilon_{32}$

where $\varepsilon_{31}$ and $\varepsilon_{32}$ are approximation errors. Choose the reaching law $\dot{S}_{3}=-k_{31} S_{3}-k_{32} S_{3} /\left(\left|S_{3}\right|+\delta\right)$. Then the actual control signal $\delta_{e}$ can be determined as

$$
\begin{aligned}
\delta_{e}= & -S_{2}-k_{31} S_{3}-k_{32} S_{3} /\left(\left|S_{3}\right|+\delta\right)-\left(\hat{\theta}_{3 l}^{T} \xi_{3 l}+\hat{\theta}_{3 r}^{T} \xi_{3 r}\right) / 2 \\
& +\dot{Q}_{d}\left(\hat{\lambda}_{3 l}^{T} \psi_{3 l}+\hat{\lambda}_{3 r}^{T} \psi_{3 r}\right) / 2
\end{aligned}
$$

where $k_{31}$ and $k_{32}$ are positive designed constants; $\hat{\theta}_{3 l}, \hat{\theta}_{3 r}, \hat{\lambda}_{3 l}$, $\hat{\lambda}_{3 r}$ are estimated values of $\theta_{3 l}^{*}, \theta_{3 r}^{*}, \lambda_{3 l}^{*}, \lambda_{3 r}^{*}$ respectively. The adaptive update laws of $\hat{\theta}_{3 l}, \hat{\theta}_{3 r}, \hat{\lambda}_{3 l}, \hat{\lambda}_{3 r}$ can be written as

$$
\begin{aligned}
& \dot{\hat{\theta}}_{3 l}=\gamma_{3 l}\left(\xi_{3 l} S_{3} / 2-c_{3 l} \hat{\theta}_{3 l}\right) \\
& \dot{\hat{\theta}}_{3 r}=\gamma_{3 r}\left(\xi_{3 r} S_{3} / 2-c_{3 r} \hat{\theta}_{3 r}\right) \\
& \dot{\hat{\lambda}}_{3 l}=\tau_{3 l}\left(-\psi_{3 l} S_{3} \dot{Q}_{d} / 2-d_{3 l} \hat{\lambda}_{3 l}\right) \\
& \dot{\hat{\lambda}}_{3 r}=\tau_{3 r}\left(-\psi_{3 r} S_{3} \dot{Q}_{d} / 2-d_{3 r} \hat{\lambda}_{3 r}\right)
\end{aligned}
$$

Assumption 3: Define the fuzzy approxiamte errors as $\varepsilon_{V}=$ $\varepsilon_{V 1}-\varepsilon_{V 2} \dot{V}_{d}, \varepsilon_{1}=\varepsilon_{11}-\varepsilon_{12} \dot{\gamma}_{d}$ and $\varepsilon_{3}=\varepsilon_{31}-\varepsilon_{32} \dot{Q}_{d}$, there exists positive constants $\varepsilon_{i}^{M}$ such that $\left|\varepsilon_{i}\right| \leq \varepsilon_{i}^{M}, i=V, 1,2,3$

\subsubsection{Stability analysis}

Theorem 2: Suppose that Assumptions 2 and 3 are satisfied. Considering the whole system under the proposed control law (27) and (42), the system is uniformly stable if adaptive update laws (28),(35),(39),(43) are used.

Proof: Define the estimation errors

$\tilde{\theta}_{k}=\hat{\theta}_{k}-\theta_{k}^{*}, k=V l, V r, 1 l, 1 r, 2 l, 2 r, 3 l, 3 r$

$\tilde{\lambda}_{p}=\hat{\lambda}_{p}-\lambda_{p}^{*}, p=V l, V r, 1 l, 1 r, 3 l, 3 r$

and filter errors

$y_{2}=\alpha_{d}-\bar{\alpha}_{d}$

$y_{3}=Q_{d}-\bar{Q}_{d}$

Substituting $\phi, \alpha_{d}, Q_{d}$ and $\delta_{e}$ into $\dot{S}_{V}, \dot{S}_{1}, \dot{S}_{2}$ and $\dot{S}_{3}$ respectively, we have

$$
\begin{aligned}
\dot{S}_{V}= & g_{V}\left[-k_{V 1} S_{V}-k_{V 2} S_{V} /\left(\left|S_{V}\right|+\delta\right)-\left(\tilde{\theta}_{V l}^{T} \xi_{V l}+\tilde{\theta}_{V r}^{T} \xi_{V r}\right) / 2\right. \\
& \left.+\dot{V}_{d}\left(\tilde{\lambda}_{V l}^{T} \psi_{V l}+\tilde{\lambda}_{V r}^{T} \psi_{V r}\right) / 2+\varepsilon_{V}\right] \\
\dot{S}_{1}= & g_{1}\left[S_{2}+y_{2}-k_{11} S_{1}-k_{12} S_{1} /\left(\left|S_{1}\right|+\delta\right)\right. \\
& \left.-\left(\tilde{\theta}_{1 l}^{T} \xi_{1 l}+\tilde{\theta}_{1 r}^{T} \xi_{1 r}\right) / 2+\dot{\gamma}_{d}\left(\tilde{\lambda}_{1 l}^{T} \psi_{1 l}+\tilde{\lambda}_{1 r}^{T} \psi_{1 r}\right) / 2+\varepsilon_{1}\right] \\
\dot{S}_{2}= & S_{3}+y_{3}-S_{1}-k_{21} S_{2}-k_{22} S_{2} /\left(\left|S_{2}\right|+\delta\right) \\
& -\left(\tilde{\theta}_{2 l}^{T} \xi_{2 l}+\tilde{\theta}_{2 r}^{T} \xi_{2 r}\right) / 2+\varepsilon_{2} \\
\dot{S}_{3}= & g_{3}\left[-S_{2}-k_{31} S_{3}-k_{32} S_{3} /\left(\left|S_{3}\right|+\delta\right)-\left(\tilde{\theta}_{3 l}^{T} \xi_{3 l}+\tilde{\theta}_{3 r}^{T} \xi_{3 r}\right) / 2\right. \\
& \left.+\dot{Q}_{d}\left(\tilde{\lambda}_{3 l}^{T} \psi_{3 l}+\tilde{\lambda}_{3 r}^{T} \psi_{3 r}\right) / 2+\varepsilon_{3}\right]
\end{aligned}
$$

besides

$\dot{y}_{i}=-\frac{y_{i}}{\kappa_{i}}+B_{i}(\cdot) \quad i=2,3$

$B_{2}(\cdot)=-\dot{\bar{\alpha}}_{d}$

$B_{3}(\cdot)=-\dot{\bar{Q}}_{d}$

Assumption 5: The FPA reference signal $\gamma_{d}$ and its derivatives $\left(\dot{\gamma}_{d}, \ddot{\gamma}_{d}\right)$ are smooth and bounded functions.

According to Assumption 5, we know there exist constants $M_{i}>0, i=2,3$ with

$\left|B_{i}(\cdot)\right| \leq M_{i}$

Consider the following Lyapunov function candidate

$W=W_{V}+W_{1}+W_{2}+W_{3}$

where

$$
\begin{aligned}
& W_{V}=\frac{S_{V}^{2}}{2 g_{V}}+\frac{\left\|\tilde{\theta}_{V l}\right\|^{2}}{2 \gamma_{V l}}+\frac{\left\|\tilde{\theta}_{V r}\right\|^{2}}{2 \gamma_{V r}}+\frac{\left\|\tilde{\lambda}_{V l}\right\|^{2}}{2 \tau_{V l}}+\frac{\left\|\tilde{\lambda}_{V r}\right\|^{2}}{2 \tau_{V r}} \\
& W_{1}=\frac{S_{1}^{2}}{2 g_{1}}+\frac{\left\|\tilde{\theta}_{1 l}\right\|^{2}}{2 \gamma_{1 l}}+\frac{\left\|\tilde{\theta}_{1 r}\right\|^{2}}{2 \gamma_{1 r}}+\frac{\left\|\tilde{\lambda}_{1 l}\right\|^{2}}{2 \tau_{1 l}}+\frac{\left\|\tilde{\lambda}_{1 r}\right\|^{2}}{2 \tau_{1 r}}+\frac{1}{2} y_{2}^{2} \\
& W_{2}=\frac{S_{2}^{2}}{2}+\frac{\left\|\tilde{\theta}_{2 l}\right\|^{2}}{2 \gamma_{2 l}}+\frac{\left\|\tilde{\theta}_{2 r}\right\|^{2}}{2 \gamma_{2 r}}+\frac{1}{2} y_{3}^{2} \\
& W_{3}=\frac{S_{3}^{2}}{2 g_{3}}+\frac{\left\|\tilde{\theta}_{3 l}\right\|^{2}}{2 \gamma_{3 l}}+\frac{\left\|\tilde{\theta}_{3 r}\right\|^{2}}{2 \gamma_{3 r}}+\frac{\left\|\tilde{\lambda}_{3 l}\right\|^{2}}{2 \tau_{3 l}}+\frac{\left\|\tilde{\lambda}_{3 r}\right\|^{2}}{2 \tau_{3 r}}
\end{aligned}
$$

Substituting $\dot{S}_{V}$ into the derivative of $W_{V}$, we can obtain

$$
\begin{aligned}
\dot{W}_{V}= & \frac{1}{g_{V}} S_{V} \dot{S}_{V}-\frac{\dot{g}_{V}}{2 g_{V}^{2}} S_{V}^{2}+\frac{1}{\gamma_{V l}} \tilde{\theta}_{V l}^{T} \dot{\hat{\theta}}_{V l}+\frac{1}{\gamma_{V r}} \tilde{\theta}_{V r}^{T} \dot{\hat{\theta}}_{V r} \\
& +\frac{1}{\tau_{V l}} \tilde{\lambda}_{V l}^{T} \dot{\hat{\lambda}}_{V l}+\frac{1}{\tau_{V r}} \tilde{\lambda}_{V r}^{T} \dot{\hat{\hat{\lambda}}}_{V r} \\
= & -k_{V 1} S_{V}^{2}-\frac{\dot{g}_{V}}{2 g_{V}^{2}} S_{V}^{2}-k_{V 2} \frac{S_{V}^{2}}{\left|S_{V}\right|+\delta}+S_{V} \varepsilon_{V} \\
& +\tilde{\theta}_{V l}^{T}\left(-\frac{\xi_{V l} S_{V}}{2}+\frac{\dot{\hat{\theta}}_{V l}}{\gamma_{V l}}\right)+\tilde{\theta}_{V r}^{T}\left(-\frac{\xi_{V r} S_{V}}{2}+\frac{\dot{\hat{\theta}}_{V r}}{\gamma_{V r}}\right) \\
& +\tilde{\lambda}_{V l}^{T}\left(\frac{\psi_{V l} S_{V} \dot{V}_{d}}{2}+\frac{\dot{\hat{\lambda}}_{V l}}{\tau_{V l}}\right)+\tilde{\lambda}_{V r}^{T}\left(\frac{\psi_{V r} S_{V} \dot{V}_{d}}{2}+\frac{\dot{\hat{\lambda}}_{V r}}{\tau_{V r}}\right)
\end{aligned}
$$


and noticing

$$
\begin{aligned}
& -\left(k_{V 1}+\frac{\dot{g}_{V}}{2 g_{V}^{2}}\right) S_{V}^{2} \leq-\left(k_{V 1}-\frac{g_{V}^{d}}{2 g_{V}^{2}}\right) S_{V}^{2}=-k_{V 1}^{*} S_{V}^{2} \\
& -k_{V 2} \frac{S_{V}^{2}}{\left|S_{V}\right|+\delta}+S_{V} \varepsilon_{V} \leq \frac{\left(\left|S_{V}\right|+\delta\right)\left(\varepsilon_{V}^{M}\right)^{2}}{4 k_{V 2}}
\end{aligned}
$$

where $k_{V 1}^{*}=k_{V 1}-g_{V}^{d} / 2 g_{V}^{2}>0$. Subsituting (28) and (52) into (51), and noticing

$$
\begin{aligned}
& -c_{V l} \tilde{\theta}_{V l}^{T} \hat{\theta}_{V l} \leq-\frac{c_{V l}\left\|\tilde{\theta}_{V l}\right\|^{2}}{2}+\frac{c_{V l}\left\|\theta_{V l}^{*}\right\|^{2}}{2} \\
& -c_{V r} \tilde{\theta}_{V r}^{T} \hat{\theta}_{V r} \leq-\frac{c_{V r}\left\|\tilde{\theta}_{V r}\right\|^{2}}{2}+\frac{c_{V r}\left\|\theta_{V r}^{*}\right\|^{2}}{2} \\
& -d_{V l} \tilde{\lambda}_{V l}^{T} \hat{\lambda}_{V l} \leq-\frac{d_{V l}\left\|\tilde{\lambda}_{V l}\right\|^{2}}{2}+\frac{d_{V l}\left\|\lambda_{V l}^{*}\right\|^{2}}{2} \\
& -d_{V r} \tilde{\lambda}_{V r}^{T} \hat{\lambda}_{V r} \leq-\frac{d_{V r}\left\|\tilde{\lambda}_{V r}\right\|^{2}}{2}+\frac{d_{V r}\left\|\lambda_{V r}^{*}\right\|^{2}}{2}
\end{aligned}
$$

Then (51) becomes

$$
\begin{gathered}
\dot{W}_{V} \leq-k_{V 1}^{*} S_{V}^{2}+\frac{\left(\left|S_{V}\right|+\delta\right)\left(\varepsilon_{V}^{M}\right)^{2}}{4 k_{V 2}}-\frac{c_{V l}\left\|\tilde{\theta}_{V l}\right\|^{2}}{2}+\frac{c_{V l}\left\|\theta_{V l}^{*}\right\|^{2}}{2} \\
-\frac{c_{V r}\left\|\tilde{\theta}_{V r}\right\|^{2}}{2}+\frac{c_{V r}\left\|\theta_{V r}^{*}\right\|^{2}}{2}-\frac{d_{V l}\left\|\tilde{\lambda}_{V l}\right\|^{2}}{2} \\
+\frac{d_{V l}\left\|\lambda_{V l}^{*}\right\|^{2}}{2}-\frac{d_{V r}\left\|\tilde{\lambda}_{V r}\right\|^{2}}{2}+\frac{d_{V r}\left\|\lambda_{V r}^{*}\right\|^{2}}{2}
\end{gathered}
$$

The time derivative of $W_{1}$ along the system trajectories is:

$$
\begin{aligned}
\dot{W}_{1} \leq & S_{1} S_{2}+S_{1} y_{2}-k_{11}^{*} S_{1}^{2}+\frac{\left(\left|S_{1}\right|+\delta\right)\left(\varepsilon_{1}^{M}\right)^{2}}{4 k_{12}}-\frac{y_{2}^{2}}{\kappa_{2}}+y_{2} B_{2} \\
& -\frac{c_{1 l}\left\|\tilde{\theta}_{1 l}\right\|^{2}}{2}+\frac{c_{1 l}\left\|\theta_{1 l}^{*}\right\|^{2}}{2}-\frac{c_{1 r}\left\|\tilde{\theta}_{1 r}\right\|^{2}}{2}+\frac{c_{1 r}\left\|\theta_{1 r}^{*}\right\|^{2}}{2} \\
& -\frac{d_{1 l}\left\|\tilde{\lambda}_{1 l}\right\|^{2}}{2}+\frac{d_{1 l}\left\|\lambda_{1 l}^{*}\right\|^{2}}{2}-\frac{d_{1 r}\left\|\tilde{\lambda}_{1 r}\right\|^{2}}{2}+\frac{d_{1 r}\left\|\lambda_{1 r}^{*}\right\|^{2}}{2}
\end{aligned}
$$

where $k_{11}^{*}=k_{11}-g_{1}^{d} / 2 g_{1}^{2}>0$. Using Young's inequality $a b \leq$ $a^{2} / 2+b^{2} / 2$, we have

$$
\begin{aligned}
\dot{W}_{1} \leq & S_{1} S_{2}-\left(k_{11}^{*}-\frac{1}{2}\right) S_{1}^{2}+\frac{\left(\left|S_{1}\right|+\delta\right)\left(\varepsilon_{1}^{M}\right)^{2}}{4 k_{12}} \\
& -\left(\frac{1}{\kappa_{2}}-1\right) y_{2}^{2}+\frac{1}{2} M_{2}^{2}-\frac{c_{1 l}\left\|\tilde{\theta}_{1 l}\right\|^{2}}{2}+\frac{c_{1 l}\left\|\theta_{1 l}^{*}\right\|^{2}}{2} \\
& -\frac{c_{1 r}\left\|\tilde{\theta}_{1 r}\right\|^{2}}{2}+\frac{c_{1 r}\left\|\theta_{1 r}^{*}\right\|^{2}}{2}-\frac{d_{1 l}\left\|\tilde{\lambda}_{1 l}\right\|^{2}}{2}+\frac{d_{1 l}\left\|\lambda_{1 l}^{*}\right\|^{2}}{2} \\
& -\frac{d_{1 r}\left\|\tilde{\lambda}_{1 r}\right\|^{2}}{2}+\frac{d_{1 r}\left\|\lambda_{1 r}^{*}\right\|^{2}}{2}
\end{aligned}
$$

where $k_{11}^{*}-1 / 2>0,1 / \kappa_{2}-1>0$. Similarly, we can get

$$
\begin{aligned}
\dot{W}_{2} \leq & S_{2} S_{3}-S_{1} S_{2}-\left(k_{21}-\frac{1}{2}\right) S_{2}^{2}+\frac{\left(\left|S_{2}\right|+\delta\right)\left(\varepsilon_{2}^{M}\right)^{2}}{4 k_{22}} \\
& -\left(\frac{1}{\kappa_{3}}-1\right) y_{3}^{2}+\frac{1}{2} M_{3}^{2}-\frac{c_{2 l}\left\|\tilde{\theta}_{2 l}\right\|^{2}}{2}+\frac{c_{2 l}\left\|\theta_{2 l}^{*}\right\|^{2}}{2} \\
& -\frac{c_{2 r}\left\|\tilde{\theta}_{2 r}\right\|^{2}}{2}+\frac{c_{2 r}\left\|\theta_{2 r}^{*}\right\|^{2}}{2}
\end{aligned}
$$

$$
\begin{aligned}
\dot{W}_{3} \leq & -S_{2} S_{3}-k_{31}^{*} S_{1}^{2}+\frac{\left(\left|S_{3}\right|+\delta\right)\left(\varepsilon_{3}^{M}\right)^{2}}{4 k_{32}}-\frac{c_{3 l}\left\|\tilde{\theta}_{3 l}\right\|^{2}}{2} \\
& +\frac{c_{3 l}\left\|\theta_{3 l}^{*}\right\|^{2}}{2}-\frac{c_{3 r}\left\|\tilde{\theta}_{3 r}\right\|^{2}}{2}+\frac{c_{3 r}\left\|\theta_{3 r}^{*}\right\|^{2}}{2}-\frac{d_{3 l}\left\|\tilde{\lambda}_{3 l}\right\|^{2}}{2} \\
& +\frac{d_{3 l}\left\|\lambda_{3 l}^{*}\right\|^{2}}{2}-\frac{d_{3 r}\left\|\tilde{\lambda}_{3 r}\right\|^{2}}{2}+\frac{d_{3 r}\left\|\lambda_{3 r}^{*}\right\|^{2}}{2}
\end{aligned}
$$

where $k_{21}-1 / 2>0,1 / \kappa_{3}-1>0, k_{31}^{*}=k_{31}-g_{3}^{d} / 2 g_{3}^{2}>0$. Then, from (49), (54), (56), (57) and (58), we can obtain the following inequality

$\dot{W} \leq-\rho_{W} W+\delta_{W}$

where $0<\rho_{W} \leq \min \left\{2 \underline{g}_{V} k_{V 1}^{*}, 2 \underline{g}_{1}\left(k_{11}^{*}-1 / 2\right), 2\left(1 / \kappa_{2}-1\right)\right.$, $\left.2\left(k_{21}-1 / 2\right), 2\left(1 / \kappa_{3}-1\right), \underline{g}_{3} k_{31}^{*}, c_{i l} \gamma_{i l}, c_{i r} \gamma_{i r}, d_{j l} \tau_{j l}, d_{j r} \tau_{j r}\right\}$, $i=V, 1,2,3, j=V, 1,3$, and

$$
\begin{aligned}
\delta_{W}= & \frac{\left(\left|S_{V}\right|+\delta\right)\left(\varepsilon_{V}^{M}\right)^{2}}{4 k_{V 2}}+\frac{c_{V l}\left\|\theta_{V l}^{*}\right\|^{2}}{2}+\frac{c_{V r}\left\|\theta_{V r}^{*}\right\|^{2}}{2} \\
& +\sum_{i=1}^{3}\left(\frac{\left(\left|S_{i}\right|+\delta\right)\left(\varepsilon_{i}^{M}\right)^{2}}{4 k_{i 2}}+\frac{c_{i l}\left\|\theta_{i l}^{*}\right\|^{2}}{2}+\frac{c_{i r}\left\|\theta_{i r}^{*}\right\|^{2}}{2}\right) \\
& +\frac{d_{V l}\left\|\lambda_{V l}^{*}\right\|^{2}}{2}+\frac{d_{V r}\left\|\lambda_{V r}^{*}\right\|^{2}}{2}+\frac{d_{1 l}\left\|\lambda_{1 l}^{*}\right\|^{2}}{2}+\frac{d_{1 r}\left\|\lambda_{1 r}^{*}\right\|^{2}}{2} \\
& +\frac{d_{3 l}\left\|\lambda_{3 l}^{*}\right\|^{2}}{2}+\frac{d_{3 r}\left\|\lambda_{3 r}^{*}\right\|^{2}}{2}+\frac{1}{2} M_{2}^{2}+\frac{1}{2} M_{3}^{2}>0
\end{aligned}
$$

By solving (59), the following inequality is given by

$$
W(t) \leq W\left(t_{0}\right) e^{-\rho_{W}\left(t-t_{0}\right)}+\delta_{W} / \rho_{W}
$$

According to (54) and (61), we have

$$
\left|S_{V}\right| \leq \sqrt{2 g_{V} W_{V}}, \quad \lim _{t \rightarrow \infty}\left|S_{V}\right| \leq \sqrt{\frac{2 \bar{g}_{V} \delta_{V}}{\rho_{V}}}
$$

The parameters $\delta_{W}$ and $\rho_{W}$ should be chosen appropriately to make $\sqrt{2 \delta_{W} / \rho_{W}}$ as small as desired. Then the signal $S_{V}$ can be globally uniformly ultimately bounded. So does $S_{1}, S_{2}, S_{3}, \tilde{\theta}_{i l}$, $\tilde{\theta}_{i r}(i=V, 1,2,3), \tilde{\lambda}_{j l}$ and $\tilde{\lambda}_{j r}(j=V, 1,3), y_{2}$ and $y_{3}$. Thus, the control system is uniformly stable.

\section{Simulation Results}

In this section, we verify the performance of the proposed adaptive interval type- 2 fuzzy SMC. The initial state at $V(0)=$ 7.0Mach, $h(0)=75000 f t, \gamma(0)=0, \alpha(0)=0, Q(0)=0$. Considering the actual situation of AHV, the following constraints should be added: $\phi \in[0,1], \delta_{e} \in[-15,15], \gamma \in[-0.5,0.5]$, $\alpha \in[-5,15]$ and $Q \in[-2,2]$. The parameters of control system are shown in Table. 1.

The simulations are divided into three parts. The first simulation case is concerned with the effectiveness of the adaptive 
Table 1: Control Parameters

\begin{tabular}{cccc}
\hline Parameters & Values & Parameters & Values \\
\hline$k_{V 1}, k_{V 1}$ & $0.5 ; 1.2$ & $\delta$ & 0.01 \\
$k_{h}$ & 12 & $k_{11}, k_{12}$ & $15 ; 10$ \\
$k_{21}, k_{22}$ & $1.4 ; 2$ & $k_{31}, k_{32}$ & $2 ; 0.4$ \\
$\kappa_{2}, \kappa_{3}$ & $0.1 ; 0.1$ & $\gamma_{V l}, \gamma_{V r}, c_{V l}, c_{V r}$ & 0.2 \\
$\tau_{V l}, \tau_{V r}, d_{V l}, d_{V r}$ & 0.2 & $\gamma_{1 l}, \gamma_{1 r}, c_{1 l}, c_{1 r}$ & 1.5 \\
$\tau_{1 l}, \tau_{1 r}, d_{1 l}, d_{1 r}$ & 1.5 & $\gamma_{2 l}, \gamma_{2 r}, c_{2 l}, c_{2 r}$ & 1 \\
$\gamma_{3 l}, \gamma_{3 r}, c_{3 l}, c_{3 r}$ & 1 & $\tau_{3 l}, \tau_{3 r}, d_{3 l}, d_{3 r}$ & 1 \\
\hline
\end{tabular}

interval type-2 fuzzy sliding mode control with VGI at the condition of a slow acceleration process (7-7.5 Mach) by comparing with FGI using the same control method. The second case: a fast acceleration process (velocity increment is increased to 2 Mach (7-9 Mach)). The third case: the altitude increment is $2200 \mathrm{ft}$ (75000-77200 ft) and the velocity is kept at 7 Mach. In all case, a nonlinear filter is used to generate the differentiable commands as follows

$W_{V}(s)=\frac{\omega_{n 1} \omega_{n 2}^{2}}{\left(s+\omega_{n 1}\right)\left(s^{2}+2 \xi_{1} \omega_{n 2} s+\omega_{n 2}^{2}\right)}$

where $\omega_{n 1}=0.1, \omega_{n 2}=0.3, \xi_{1}=30$.

Case 1: The velocity increment is 0.5 Mach.
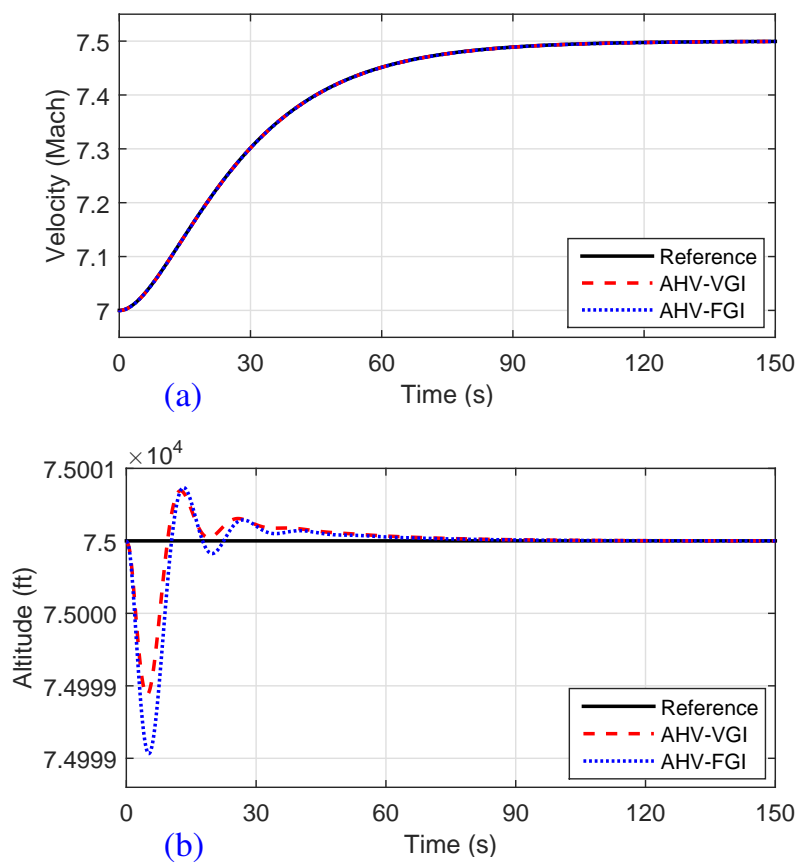

Fig. 8 and Fig. 9 show the simulation results. From Fig. 8(a), the velocity can track command signal $V_{d}$ accurately and the altitude can converge to its initial state without error. The two control systems can achieve a very good tracking performance. Compared the FER curves of AHV-FGI and AHV-VGI, there
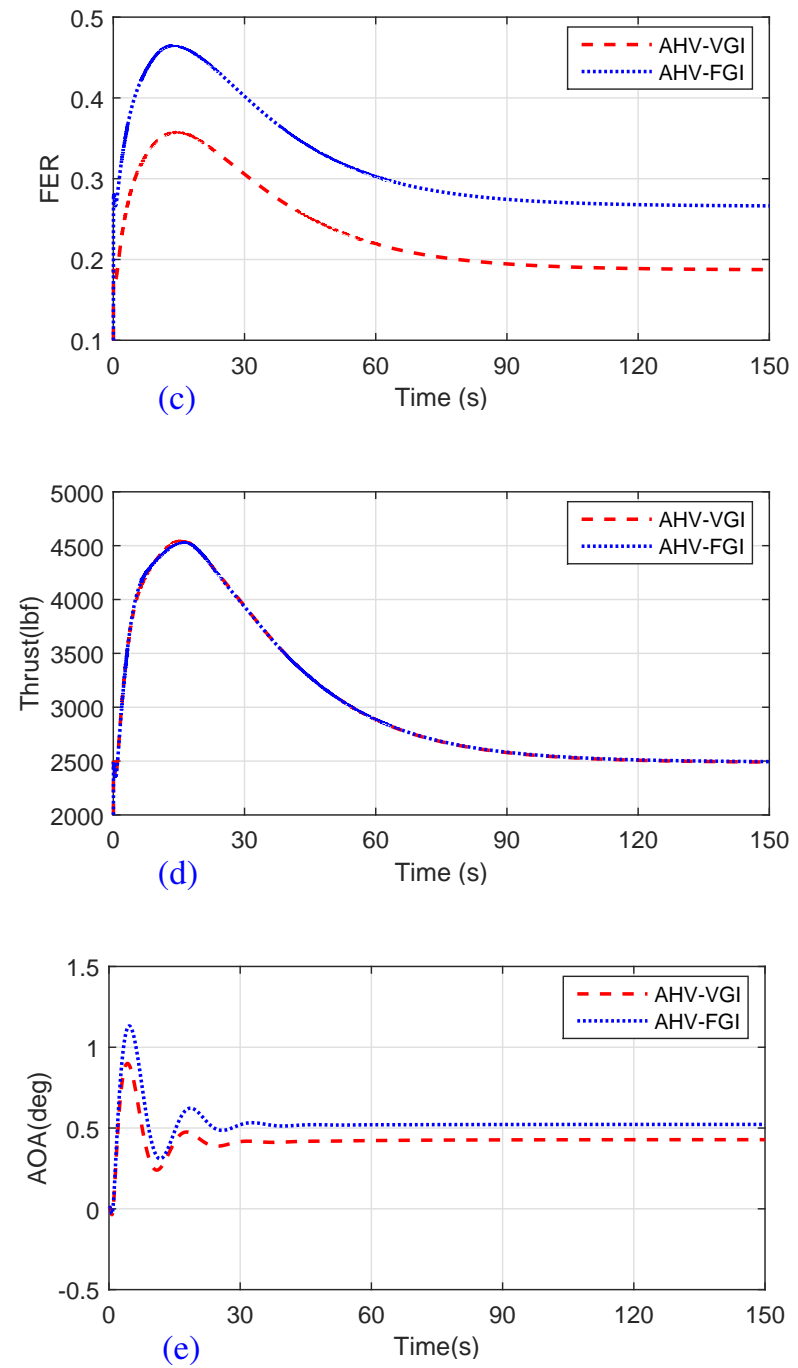

Fig. 8. Response to a 0.5 Mach velocity increment command. (a)velocity; (b)altitude; (c)FER; (d)thrust; (e)AOA

are obvious difference shown in the Fig. 8(c). When the stable situations were acquired, the FER of AHV-FGI is 0.2664, and this value is 0.1875 in AHV-VGI. As one of control inputs of AHV system, the FER $\phi$ is the control variable of the thrust $T$. As shown in Fig. 8(d), the thrust in these two control systems are almost equal. When the velocity command is given, the AHV-VGI can capture more free stream and offer greater thrust due to the existence of movable translating cowl. Therefore, the AHV-VGI can provide the required thrust with a smaller FER, which is obviously beneficial for the fuel saving. From Fig. 8(e), it is verified that AOA $\alpha$ is slightly different between AHV-VGI and AHV-FGI. That is because elongation distance $l$ induces the pitching moment changes. In order to ensure the stability of the control system, the control input $\delta_{e}$ has to be slightly adjusted, which causes the change of AOA. Of course, it does not have much impacts on the AHV flight.

The elongation diatance $l$ is shown in Fig. 9. It is gradually decreased from 9.5536 to 7.8653 . With the increasing of the velocity, the shock wave angle decreases, and the oblique shock wave generated by AHV frontbody is assembled to the body, so the movable cowl moves back. Besides, as shown in 


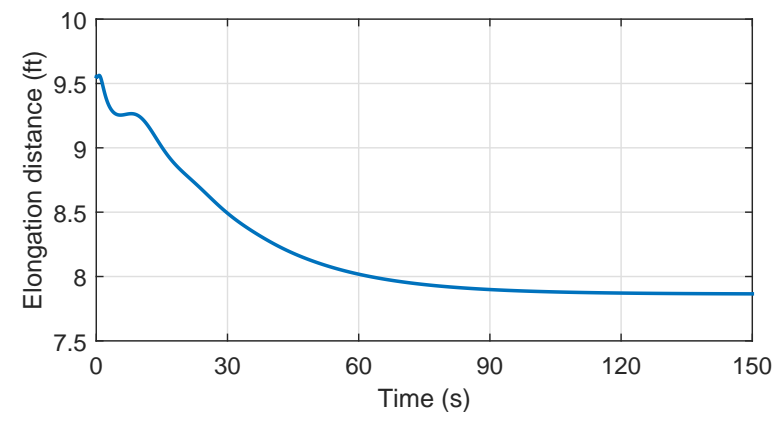

Fig. 9. The elongation distance for a $0.5 \mathrm{Ma}$ command
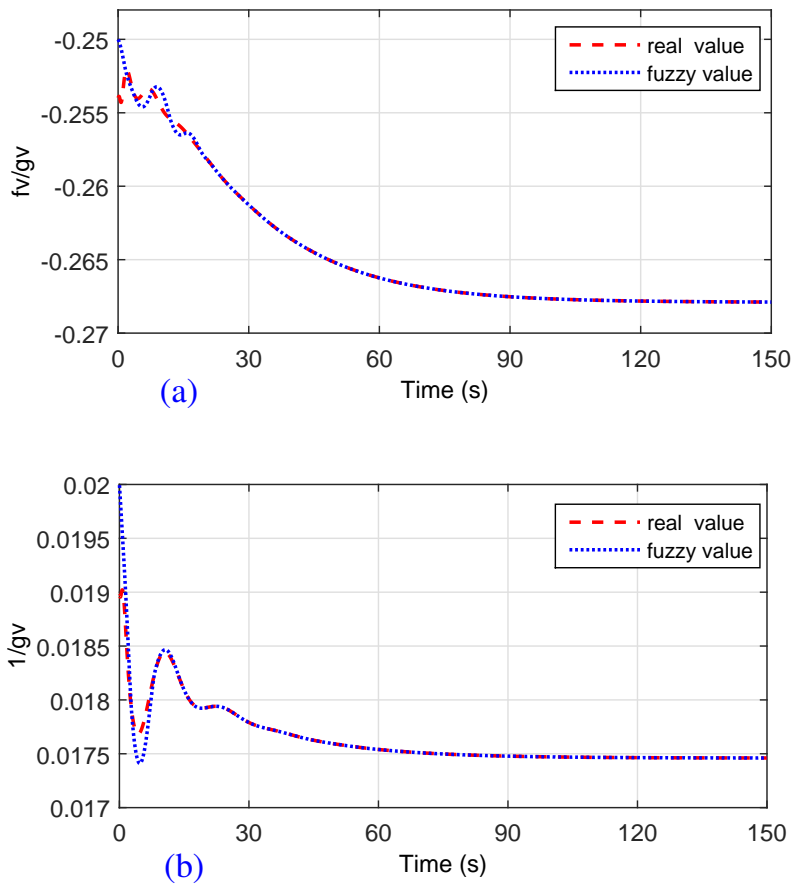

Fig. 10. The approaching effect of interval type-2 system

Fig. 10, we take equation (26) as an example to illustrate the effectiveness of the interval type- 2 system (the AHV-VGI system), which have testified the interval type- 2 system can approach its desired value rapidly.

Case 2: The velocity increment is 2 Mach. This velocity command changes quickly.

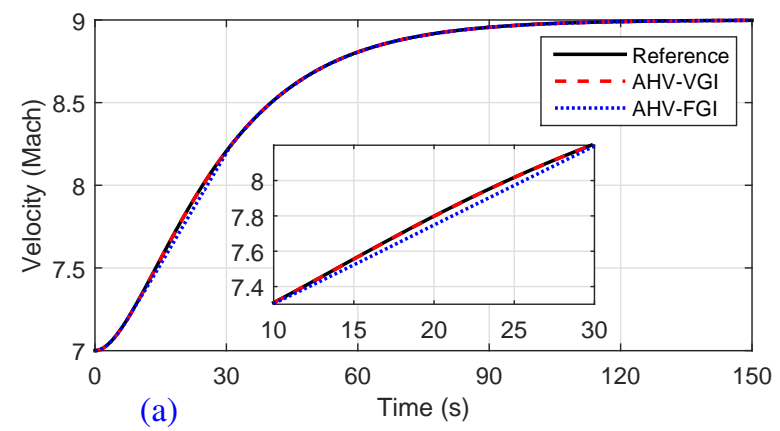

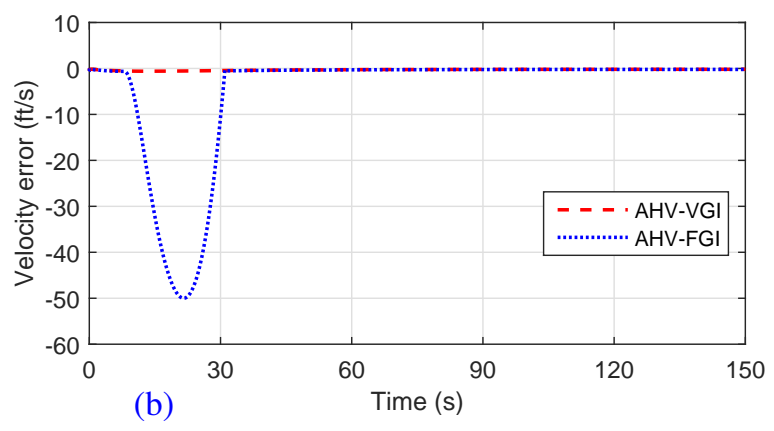
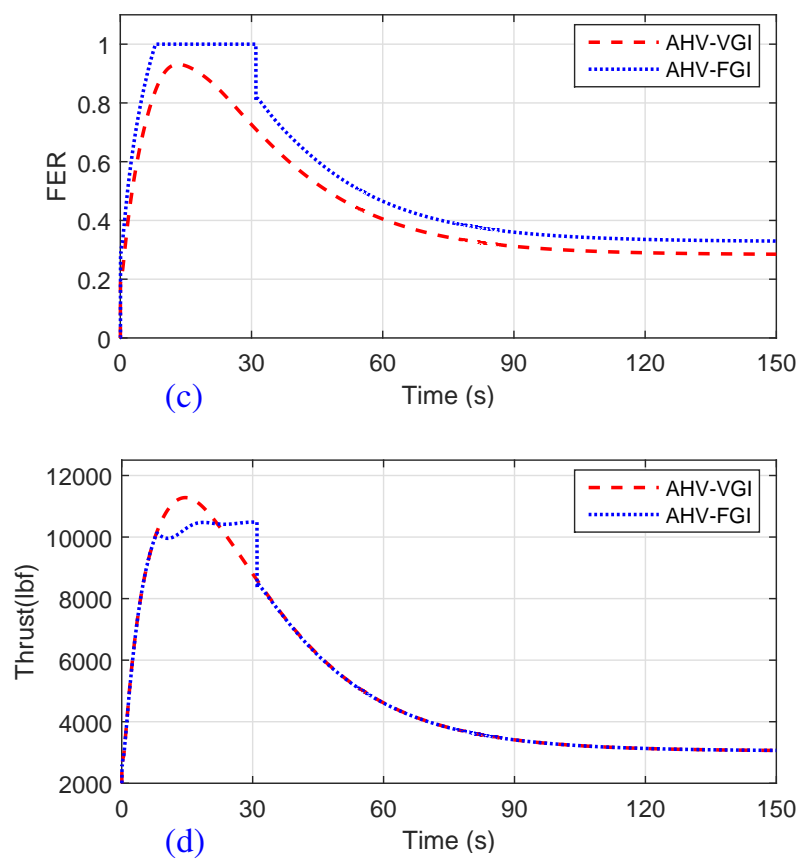

Fig. 11. Response to a 1.8Ma velocity command. (a)velocity; (b) velocity error; (c)FER; (d)Thrust

The simulation results are shown in Fig. 11, which illustrate that the AHV-VGI control system can accurately track a fast changing velocity command. In the fast acceleration process, the FER $\phi$ is large in order to provide the required thrust. However, considering $\phi \in[0,1]$, the FER of the AHV-FGI will reach its saturated state during the time of 10-20s (shown in Fig. 11 (c)), which causes the velocity tracking error increased suddenly (shown in Fig. 11 (a) (b)) and the response curve of the thrust is distorted (shown in Fig. 11(d)). In practical applications, the FER saturation may causes AHV fails to track the flight instruction, or lead to more serious consequences. However, the AHV-VGI control system can provide the required thrust by adjusting the movable cowl, and in a smaller FER value, which greatly reduces the possibility of the FER saturation(shown in Fig. 11 (c)). Hence, compared to the AHVFGI control system, the AHV-VGI control system has the better tracking performance. It is important for improving the AHV cruise and operational capability.

Case 3: The altitude increment is $2200 \mathrm{ft}$ and the velocity is kept at 7 Mach.

As shown in Fig. 12(a), the two control system can fulfill the 

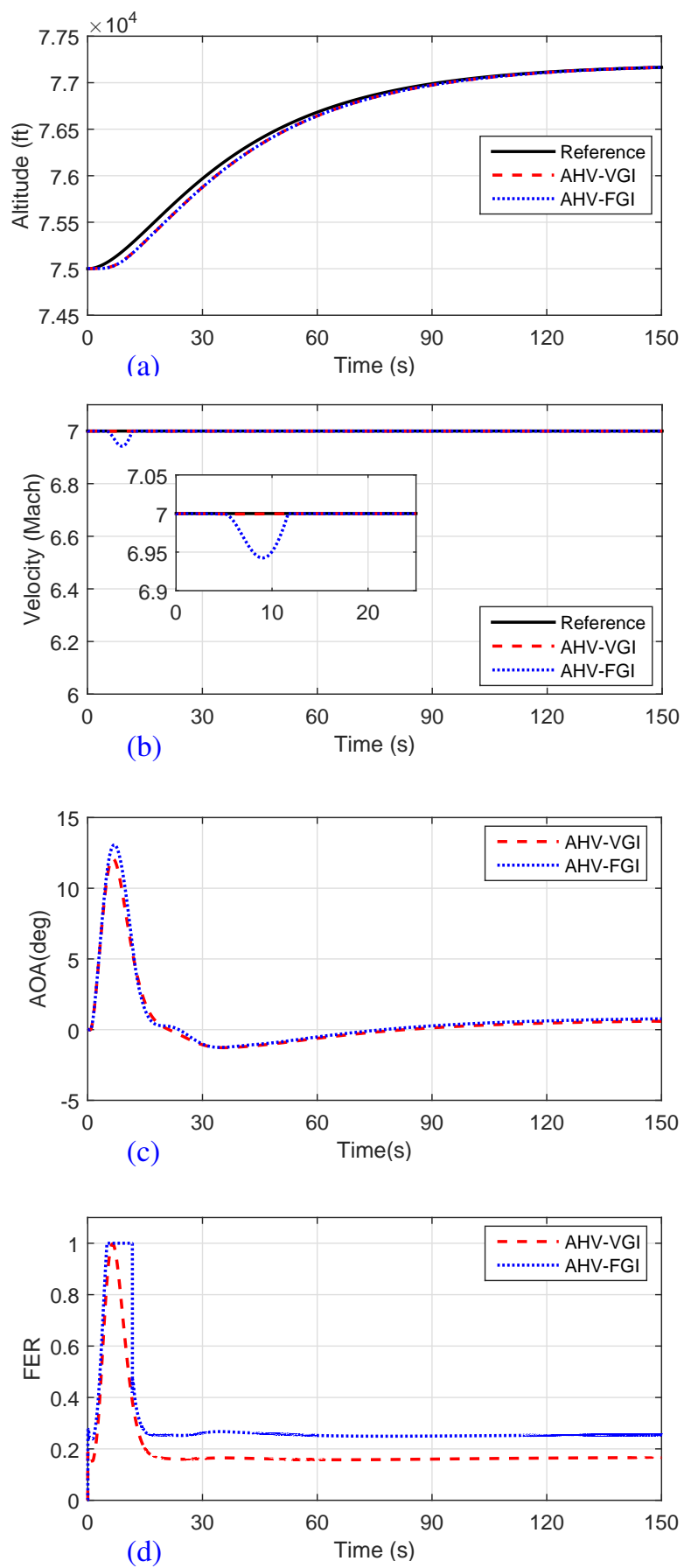

altitude tracking performance. However, the velocity happens suddenly a little error during the time $5-15 \mathrm{~s}$ in the AHV-FGI control system (shown in Fig. 12(b)). Obviously, sudden altitude increase causes the quick change of the AOA $\alpha$ (shown in Fig. 12(c)), which needs a greater thrust to ensure it. The FER $\phi$ reaches its saturated state in the AHV-FGI control system, while the AHV-VGI control system can provide the required thrust (shown in Fig. 12 (d)). Fig. 12 (e) shows the change of elongation distance $l$. Fig. 13 shows the response curve of velocity at the condition of velocity command 7-9 Mach and altitude command 75000-77200ft. On this occasion, the AHV-FGI control system can not ensure the tracking performance, while the AHV-VGI control system has a better tracking performance.

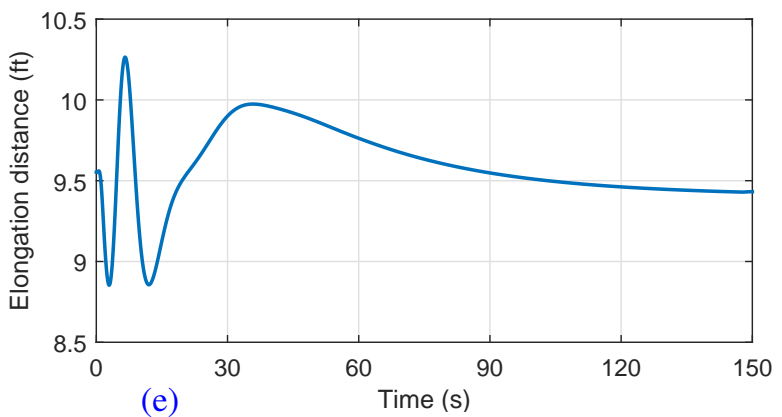

Fig. 12. Response to $2200 \mathrm{ft}$ altitude increment command. (a)altitude; (b)velocity; (c)AOA; (d)FER; (e)elongation distance

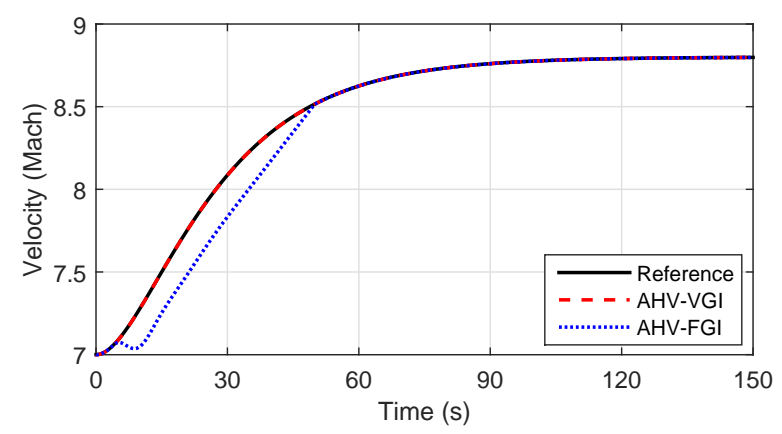

Fig. 13. Response to a velocity command 7-9 Mach and a altitude command $75000-77200 \mathrm{ft}$

\section{Conclusion}

In this paper, we proposed an adaptive interval type- 2 fuzzy sliding mode control scheme for the AHV-VGI. The simulation results shows the effectiveness of this control scheme. We illustrated the superiority of VGI in three cases. The AHV-VGI can provide required thrust and acceleration in a smaller FER compared with the traditional AHV-FGI. The using of movable translating cowl reduces the possibility of the FER saturation and extents the velocity flight range. Therefore, the research of control strategy for AHV-VGI is very vital and significant. In this paper, we assume that the translating cowl can be infinitely fast. But in fact, the cowl need be controlled suitably. Therefore, we will carry on the research on coordination control between flight control and cowl control for the AHV-VGI in further work.

\section{Appendix A.}

Table A.2: Fitting coefficients in elongation distance

\begin{tabular}{cccc}
\hline Coefficients & Values & Coefficients & Values \\
\hline$C_{l}^{\alpha}$ & -0.2804 & $C_{l}^{\alpha^{2}}$ & 0.0281 \\
$C_{l}^{M a}$ & -3.151 & $C_{l}^{0}$ & 31.611 \\
$C_{L}^{\alpha}$ & 0.0157 & $C_{L}^{M a}$ & $5.45 \mathrm{e}-05$ \\
$C_{L}^{\delta_{e}}$ & 0.0066 & $C_{L}^{0}$ & 0.0046 \\
\hline
\end{tabular}




\begin{tabular}{cccc}
\hline Coefficients & Values & Coefficients & Values \\
\hline$C_{D}^{\alpha}$ & $1.28 \mathrm{e}-04$ & $C_{D}^{\alpha^{2}}$ & $3.59 \mathrm{e}-04$ \\
$C_{D}^{\delta_{e}}$ & $-1.97 \mathrm{e}-10$ & $C_{D}^{\delta_{e}^{2}}$ & $4.37 \mathrm{e}-05$ \\
$C_{D}^{\alpha \delta_{e}}$ & $9.78 \mathrm{e}-05$ & $C_{D}^{M a}$ & $-5.32 \mathrm{e}-04$ \\
$C_{D}^{0}$ & 0.0133 & $C_{T}^{\alpha}$ & 0.0328 \\
$C_{T}^{M a}$ & 0.0026 & $C_{T}^{0}$ & -0.152 \\
$C_{T}^{\phi \alpha}$ & 0.3152 & $C_{T}^{\phi M a}$ & -0.703 \\
$C_{T}^{\phi}$ & 8.9227 & $C_{M}^{\alpha}$ & 0.0064 \\
$C_{M}^{M a}$ & -0.0022 & $C_{M}^{\delta_{e}}$ & -0.014 \\
$C_{M}^{\phi}$ & -0.0072 & $C_{M}^{0}$ & 0.051 \\
$C_{L, l}^{\alpha}$ & $9.02 \mathrm{e}-05$ & $C_{L, l}^{M a}$ & $-1.317 \mathrm{e}-04$ \\
$C_{L, l}^{0}$ & 0.0012 & $C_{D, l}^{\alpha}$ & $1.844 \mathrm{e}-05$ \\
$C_{D, l}^{M a}$ & $-1.0037-05$ & $C_{D, l}^{0}$ & $5.121 \mathrm{e}-05$ \\
$C_{T, l}^{\alpha}$ & 0.0025 & $C_{T, l}^{M a}$ & -0.0045 \\
$C_{T, l}^{0}$ & 0.0596 & $C_{M, l}^{\alpha}$ & $1.529 \mathrm{e}-05$ \\
$C_{M, l}^{M a}$ & $4.303 \mathrm{e}-05$ & $C_{M, l}^{0}$ & $-2.53 \mathrm{e}-04$ \\
\hline
\end{tabular}

[4] C. Bahm, E. Baumann, J. Martin, D. Bose, R. E. Beck, B. Strovers, The $\mathrm{X}$-43a hyper-x mach 7 flight 2 guidance, navigation, and control overview and flight test results, AIAA paper 3275 (2005) 2005.

[5] J. M. Hank, J. S. Murphy, R. C. Mutzman, The x-51a scramjet engine flight demonstration program, AIAA Paper 2540 (2008) 2008.

[6] N. Lamorte, P. P. Friedmann, D. J. Dalle, S. M. Torrez, J. F. Driscoll, Uncertainty propagation in integrated airframe-propulsion system analysis for hypersonic vehicles, Journal of Propulsion and Power 31 (1) (2014) 54-68.

[7] A. Clark, C. Wu, M. Mirmirani, S. Choi, M. Kuipers, Development of an airframe-propulsion integrated generic hypersonic vehicle model, in: The 44th AIAA Aerospace Sciences Meeting and Exhibit. Reno: AIAA, 2006, pp. 2006-218.

[8] J. D. Shaughnessy, S. Z. Pinckney, J. D. McMinn, C. I. Cruz, M.-L. Kelley, Hypersonic vehicle simulation model: winged-cone configuration, NASA TM 102610.

[9] S. Keshmiri, M. D. Mirmirani, R. Colgren, Six-dof modeling and simulation of a generic hypersonic vehicle for conceptual design studies, in: Proceedings of AIAA Modeling and Simulation Technologies Conference and Exhibit, 2004, pp. 2004-4805.

[10] R. Colgren, S. Keshmiri, M. Mirmirani, Nonlinear ten-degree-of-freedom dynamics model of a generic hypersonic vehicle, Journal of aircraft 46 (3) (2009) 800-813.

[11] C. I. Marrison, R. F. Stengel, Design of robust control systems for a hypersonic aircraft, Journal of Guidance, Control, and Dynamics 21 (1) (1998) $58-63$.

[12] Q. Wang, R. F. Stengel, Robust nonlinear control of a hypersonic aircraft, Journal of Guidance, Control, and Dynamics 23 (4) (2000) 577-585.

[13] F. R. Chavez, D. K. Schmidt, Analytical aeropropulsive-aeroelastic hypersonic-vehicle model with dynamic analysis, Journal of Guidance, Control, and Dynamics 17 (6) (1994) 1308-1319.

[14] F. R. Chavez, D. K. Schmidt, Uncertainty modeling for multivariablecontrol robustness analysis of elastic high-speed vehicles, Journal of Guidance, Control, and Dynamics 22 (1) (1999) 87-95.

[15] H. Sun, Z. Yang, J. Zeng, New tracking-control strategy for airbreathing hypersonic vehicles, Journal of Guidance, Control, and Dynamics 36 (3) (2013) 846-859.

[16] M. A. Bolender, D. B. Doman, A non-linear model for the longitudinal dynamics of a hypersonic air-breathing vehicle, AIAA paper 6255 (2005) 2005.

[17] J. T. Parker, A. Serrani, S. Yurkovich, M. A. Bolender, D. B. Doman, Control-oriented modeling of an air-breathing hypersonic vehicle, Journal of Guidance, Control, and Dynamics 30 (3) (2007) 856-869.

[18] G. A. Dukeman, Profile-following entry guidance using linear quadratic regulator theory, AIAA paper 4457 (2002) 5-8.

[19] K. M. Ryan, M. J. Lewis, K. H. Yu, Comparison of robust optimization methods applied to hypersonic vehicle design, Journal of Aircraft 52 (5) (2015) 1510-1523.

[20] D. P. Wiese, A. M. Annaswamy, J. A. Muse, M. A. Bolender, Adaptive control of a generic hypersonic vehicle, in: AIAA Guidance, Navigation, and Control (GNC) Conference, 2013, p. 4514.

[21] B. Tian, Z. Zuo, X. Yan, H. Wang, A fixed-time output feedback control scheme for double integrator systems, Automatica 80 (2017) 17-24.

[22] B. Tian, W. Fan, R. Su, Q. Zong, Real-time trajectory and attitude coordination control for reusable launch vehicle in reentry phase, IEEE Transactions on Industrial Electronics 62 (3) (2015) 1639-1650.

[23] Q. Zong, J. Wang, B. Tian, Y. Tao, Quasi-continuous high-order sliding mode controller and observer design for flexible hypersonic vehicle, Aerospace Science and Technology 27 (1) (2013) 127-137.

[24] C. Mu, C. Sun, W. Xu, Fast sliding mode control on air-breathing hypersonic vehicles with transient response analysis, Proceedings of the Institution of Mechanical Engineers, Part I: Journal of Systems and Control Engineering 230 (1) (2016) 23-34.

[25] H. An, J. Liu, C. Wang, L. Wu, Disturbance observer-based antiwindup control for air-breathing hypersonic vehicles, IEEE Transactions on Industrial Electronics 63 (5) (2016) 3038-3049.

[26] C. Mu, Z. Ni, C. Sun, H. He, Air-breathing hypersonic vehicle tracking control based on adaptive dynamic programming, IEEE transactions on neural networks and learning systems 28 (3) (2017) 584-598.

[27] C. Bai, J. Chen, Z. Ren, Q. Li, Z. Xiong, Adaptive decoupling control of hypersonic vehicle using fuzzy-neural network observer, Proceedings 
of the Institution of Mechanical Engineers, Part G: Journal of Aerospace Engineering 230 (7) (2016) 1216-1223.

[28] S. O. Macheret, M. N. Shneider, R. B. Miles, Scramjet inlet control by off-body energy addition: a virtual cowl, AIAA journal 42 (11) (2004) 2294-2302.

[29] L. vonEggers Rudd, F. Rankins, D. J. Pines, Moveable cowl control for in-creased hypersonic performance, in: 8th AIAA International Space Planes and Hypersonic Systems and Technologies Conference, 1998, pp. $1-6$.

[30] L. D. Huebner, K. E. Rock, E. G. Ruf, D. W. Witte, E. H. Andrews, Hyper$\mathrm{x}$ flight engine ground testing for flight risk reduction, Journal of spacecraft and rockets 38 (6) (2001) 844-852.

[31] D. J. Dalle, S. M. Torrez, J. F. Driscoll, Performance analysis of variablegeometry scramjet inlets using a low-order model, in: University of Michigan, Ann Arbor, 47th AIAA/ASME/SAE/ASEE Joint propulsion Conference \& Exhibit, Vol. 31, 2011.

[32] T. Kojima, N. Tanatsugu, T. Sato, M. Kanda, Y. Enomoto, Development study on axisymmetric air inlet for atrex engine, in: 10th AIAA/NALNASDA-ISAS International Space Planes and Hypersonic Systems and Technologies Conference 2001, 2001.

[33] A. Kuranov, E. Sheikin, Mhd control by external and internal flow s in scramjet under ajax concept, in: 41st AIAA Aerospace Sciences Meeting-Weakly Ionized Gas Workshop, Reno, NV, AIAA Paper, Vol. 173, 2003.

[34] N. O. P. Raj, K. Venkatasubbaiah, A new approach for the design of hypersonic scramjet inlets, Physics of Fluids (1994-present) 24 (8) (2012) 086103.

[35] L. Xie, R. Guo, Numerical simulation and experimental validation of flow in mixed-compression axisymmetric supersonic inlet with fixedgeometry, Acta Aeronautica et Astronautica Sinica-series A and B- 28 (1) (2007) 78.

[36] Z. Jin, K. Zhang, W. Chen, Y. Liu, Design and regulation of twodimensional variable geometry hypersonic inlets, Acta Aeronautica et Astronautica Sinica 34 (4) (2013) 779-786.

[37] M. A. Bolender, D. B. Doman, Nonlinear longitudinal dynamical model of an air-breathing hypersonic vehicle, Journal of Spacecraft and Rockets 44 (2) (2007) 374-387.

[38] Z. Jin, K. Zhang, A variable geometry scramjet inlet with a translating cowl operating in a large mach number range, Journal of Astronautics 5 (2010) 041 .

[39] L. Dou, Y. Shen, R. Ji, Analysis of the impact of translating cowl on hypersonic vehicle performance, in: The 26th Chinese Control and Decision Conference (2014 CCDC), IEEE, 2014, pp. 3834-3839.

[40] J. M. Mendel, F. Liu, Super-exponential convergence of the karnikmendel algorithms for computing the centroid of an interval type-2 fuzzy set, IEEE Transactions on Fuzzy Systems 15 (2) (2007) 309-320. 